Comment. Math. Helv. 79 (2004) 618-646

0010-2571/04/030618-29

DOI $10.1007 / \mathrm{s} 00014-004-0816-y$ (c) 2004 Birkhäuser Verlag, Basel

Commentarii Mathematici Helvetici

\title{
Rohlin's invariant and gauge theory, I. Homology 3-tori
}

Daniel Ruberman and Nikolai Saveliev

\begin{abstract}
This is the first in a series of papers exploring the relationship between the Rohlin invariant and gauge theory. We discuss a Casson-type invariant of a 3-manifold $Y$ with the integral homology of the 3-torus, given by counting projectively flat $U(2)$-connections. We show that its mod 2 evaluation is given by the triple cup product in cohomology, and so it coincides with a certain sum of Rohlin invariants of $Y$. Our counting argument makes use of a natural action of $H^{1}\left(Y ; \mathbb{Z}_{2}\right)$ on the moduli space of projectively flat connections; along the way we construct perturbations that are equivariant with respect to this action. Combined with the Floer exact triangle, this gives a purely gauge-theoretic proof that Casson's homology sphere invariant reduces mod 2 to the Rohlin invariant.
\end{abstract}

Mathematics Subject Classification (2000). 57M27, 57R58, 58D27.

Keywords. Casson invariant, Rohlin invariant, Floer homology, flat moduli spaces.

\section{Introduction}

Casson's introduction of his invariant for homology 3 -spheres $[1,19]$ has had many profound consequences in low-dimensional topology. One of the most important is the vanishing of the Rohlin invariant of a homotopy sphere, which follows from Casson's identification of his invariant, modulo 2, with the Rohlin invariant of an arbitrary homology sphere. The proof of this identification proceeds via a surgery argument, in which a series of invariants is defined for knots and links of several components. Ultimately, these invariants are related to classical knot invariants, such as the Alexander polynomial, and the theorem follows. This surgery point of view, further developed in $[22,14]$, finds its ultimate expression in the theory of finite-type invariants of 3-manifolds [13, 16].

In this paper we give a proof of the equality of Rohlin's and Casson's invariants (modulo 2) in terms of the gauge theoretic framework introduced by Taubes [20]. Many of the ingredients for this proof are already in place, namely Taubes' original work, and the surgery sequence of Floer $[9,3]$ relating Casson-type invariants of

The first author was partially supported by NSF Grants 9971802 and 0204386 . The second author was partially supported by NSF Grant 0196523. 
manifolds obtained by surgery on a knot. Our main contribution is to identify a Casson-type invariant of a homology 3 -torus, $Y$, with a Rohlin-type invariant. This is accomplished by relating the action of the group $H^{1}\left(Y ; \mathbb{Z}_{2}\right)$ on the moduli space of (projectively) flat connections to the cup product in cohomology. The techniques we develop to deal with equivariant aspects of non-smooth moduli spaces should be of independent interest in the study of instanton Floer homology (compare [7, $\S 5.6]$ ). We have made use of the perturbation theory and other techniques from this paper in subsequent work $[17,18]$.

Let us briefly describe the invariants in question; more details will be given in the next section. By homology 3-torus we mean a closed oriented 3-manifold $Y$ having the integral homology of the 3 -torus $T^{3}=S^{1} \times S^{1} \times S^{1}$. For any nontrivial $w \in H^{2}\left(Y ; \mathbb{Z}_{2}\right)$, we consider projectively flat connections on a principal $U(2)$-bundle $P \rightarrow Y$ whose associated $S O(3)=P U(2)$-bundle has second StiefelWhitney class equal to $w$. We define a Casson-type invariant $\lambda^{\prime \prime \prime}(Y, w)$ to be one-half of the signed count of such connections, modulo an appropriate gauge group. This invariant is one-half of the Euler characteristic of the Floer homology studied in [7] and [3] and is not, a priori, an integer.

A pair consisting of a closed oriented 3-manifold $X$ and a spin structure $\sigma$ has a Rohlin invariant $\rho(X, \sigma) \in \mathbb{Q} / 2 \mathbb{Z}$. By definition,

$$
\rho(X, \sigma)=\frac{1}{8} \operatorname{sign}(V)
$$

for any spin 4-manifold $V$ with (spin) boundary $(X, \sigma)$. By the Rohlin invariant $\rho^{\prime \prime \prime}(Y)$ smooth compact of a homology 3-torus $Y$ we mean the sum, over the eight spin structures on $Y$, of their Rohlin invariants. It is easy to see that, as for a homology sphere, this invariant actually takes values in $\mathbb{Z} / 2 \mathbb{Z}$.

Theorem 1.1. For any choice of non-trivial $w \in H^{2}\left(Y ; \mathbb{Z}_{2}\right)$, the Casson invariant $\lambda^{\prime \prime \prime}(Y, w)$ is an integer. If $\left\{a_{1}, a_{2}, a_{3}\right\}$ is a basis in $H^{1}(Y ; \mathbb{Z})$ then

$$
\lambda^{\prime \prime \prime}(Y, w)=\left(a_{1} \cup a_{2} \cup a_{3}\right)[Y](\bmod 2) .
$$

Note that this implies that $\lambda^{\prime \prime \prime}(Y, w)(\bmod 2)$ is independent of $w$, so long as $w$ is non-trivial. It is a theorem of V. Turaev [21], based on S. Kaplan [12, Lemma $6.3]$ that the above triple cup product also evaluates the Rohlin invariant. Hence we obtain the following result.

Corollary 1.2. If $Y$ is a homology 3-torus, then $\lambda^{\prime \prime \prime}(Y, w) \equiv \rho^{\prime \prime \prime}(Y)(\bmod 2)$.

At the end of the paper, we will explain how this implies Casson's original result about the Rohlin invariant of homology spheres.

We conjecture that the congruence in Theorem 1.1 lifts to the integers as $\lambda^{\prime \prime \prime}(Y, w)= \pm\left(\left(a_{1} \cup a_{2} \cup a_{3}\right)[Y]\right)^{2}$. This is suggested by Casson's original work [1, 19], and a closely related formula is given by Lescop [14]. To prove this conjecture, one would have to show that the count of flat connections on a homology 3-torus 
equals either Lescop's invariant or Casson's invariant for 3-component links (of trivial linking numbers). This equality is closely related to Casson's formula for his knot invariant in terms of the Alexander polynomial; a purely gauge-theoretic proof of the latter has been given by Donaldson [6]. The techniques in the present paper are rather different, and moreover have the advantage of extending to the 4-dimensional situation [18] where the integral version does not hold.

The idea of the proof of Theorem 1.1 is to take advantage of a natural $H^{1}\left(Y ; \mathbb{Z}_{2}\right)$ $=\left(\mathbb{Z}_{2}\right)^{3}$ action on the moduli space of projectively flat connections. We identify this moduli space with the space of projective representations of $\pi_{1} Y$ in $S U(2)$, and use this identification to show that the orbits with two elements are always nondegenerate and that the number of such orbits equals $\left(a_{1} \cup a_{2} \cup a_{3}\right)[Y](\bmod 2)$. In the non-degenerate situation, this completes the proof because there are no orbits with just one element, and the orbits with four and eight elements do not contribute to $\lambda^{\prime \prime \prime}(Y, w)(\bmod 2)$. The general case reduces to the non-degenerate one after one finds a generic perturbation which is $H^{1}\left(Y ; \mathbb{Z}_{2}\right)$-equivariant. As mentioned above, this equivariance is rather delicate.

The authors thank Christopher Herald for sharing his expertise.

\section{The invariant $\lambda^{\prime \prime \prime}$}

In this section we introduce the invariant $\lambda^{\prime \prime \prime}$ of a homology 3-torus $Y$ by counting projectively flat connections in a $U(2)$-bundle over $Y$. The 'derivative' notation comes from Casson's original approach, in which $\lambda^{\prime \prime \prime}$ appears as the third difference quotient of his homology sphere invariant.

\subsection{The bundles}

Let $Y$ be a homology 3-torus, $P$ a principal $U(2)$-bundle over $Y$, and $\bar{P}$ its associated $S O(3)=P U(2)$-bundle. Topologically, the bundles $P$ and $\bar{P}$ are determined by their characteristic classes $c_{1}(P)$ and $w_{2}(\bar{P})$, which are related by the formula $w_{2}(\bar{P})=c_{1}(P)(\bmod 2)$. Since $H^{3}(Y ; \mathbb{Z})$ is torsion free, every $S O(3)$-bundle over $Y$ arises as $\bar{P}$ for some $U(2)$-bundle $P$, and $S O(3)$-bundles with non-trivial $w_{2}$ correspond to $U(2)$-bundles whose $c_{1}$ is an odd element in $H^{2}(Y ; \mathbb{Z})$.

Every connection $A$ on $P$ induces connections on $\bar{P}$ and on the line bundle $\operatorname{det} P$, via the splitting $\mathfrak{u}(2)=\mathfrak{s u}(2) \oplus \mathfrak{u}(1)$. In a local trivialization, this corresponds to the decomposition

$$
A=\left(A-\frac{1}{2} \operatorname{tr} A \cdot \mathrm{Id}\right)+\frac{1}{2} \operatorname{tr} A \cdot \mathrm{Id} .
$$

The induced connection on $\bar{P}$ is the image of the first summand under the isomorphism ad $: \mathfrak{s u}(2) \rightarrow \mathfrak{s o}(3)$ given by $\operatorname{ad}(\xi)(\eta)=[\xi, \eta]$, and the induced connection on $\operatorname{det} P$ is $\operatorname{tr} A$. Conversely, any two connections on $\bar{P}$ and $\operatorname{det} P \operatorname{determine}$ a 
unique connection on $P$.

Fix a connection $C$ on $\operatorname{det} P$, and let $\mathcal{A}(P)$ be the space of connections on $P$ compatible with $C$. The connection $C$ plays no real geometric role - different choices will give equivalent theories. The gauge group $\mathcal{G}(P)$ consisting of unitary automorphisms of $P$ of determinant one preserves $C$ and hence acts on $\mathcal{A}(P)$ with the quotient space $\mathcal{B}(P)=\mathcal{A}(P) / \mathcal{G}(P)$. Let $\mathcal{A}(\bar{P})$ be the affine space of connections on $\bar{P}$ and $\mathcal{G}(\bar{P})$ the $S O(3)$-gauge group. Denote $\mathcal{B}(\bar{P})=\mathcal{A}(\bar{P}) / \mathcal{G}(\bar{P})$. The projection $\pi: \mathcal{A}(P) \rightarrow \mathcal{A}(\bar{P})$ induced by the splitting (1) commutes with the above gauge group actions and hence defines a projection

$$
\pi: \mathcal{B}(P) \rightarrow \mathcal{B}(\bar{P})
$$

The group $H^{1}\left(Y ; \mathbb{Z}_{2}\right)$ acts on $\mathcal{B}(P)$ as follows, compare with [7, pages 146-149]. Let us view $\chi \in H^{1}\left(Y ; \mathbb{Z}_{2}\right)$ as a homomorphism from $\pi_{1} Y$ to $\mathbb{Z}_{2}=\{ \pm 1\}$. As such, it defines a flat complex line bundle $L_{\chi}$. Since $\chi$ lifts to an integral homology class, the bundle $L_{\chi}$ is trivial and hence the bundles $P$ and $P \otimes L_{\chi}$ are isomorphic. For any $A \in \mathcal{A}(P)$, let $A \otimes \chi$ be the connection on $P \otimes L_{\chi}$ induced by $A$ and $\chi$. It can be written as $A+i a \cdot \mathrm{Id}$, where $a$ is a closed real-valued 1-form. Since the connections $A$ and $A \otimes \chi$ define the same connection $C$ on $\operatorname{det}(P)=\operatorname{det}\left(P \otimes L_{\chi}\right)$, we have a well defined action on gauge equivalence classes given by the formula $\chi(A)=A \otimes \chi$.

Proposition 2.1. The map $\pi$ defined in $(2)$ is the quotient map of the $H^{1}\left(Y ; \mathbb{Z}_{2}\right)$ action described above.

Proof. The connections on $\bar{P}$ induced by $A$ and $A \otimes \chi$ are $S O(3)$-gauge equivalent because they have the same holonomy. Every connection on $\bar{P}$ arises from a connection on $P$ which is unique up to the action in question.

\subsection{Projectively flat connections}

Let $A$ be a connection on $P$ compatible with the connection $C$ on $\operatorname{det} P$ and let $\bar{A}=\pi(A)$. The projection $\pi: \mathcal{A}(P) \rightarrow \mathcal{A}(\bar{P})$ identifies the tangent spaces of $\mathcal{A}(P)$ and $\mathcal{A}(\bar{P})$ at $A$ and $\bar{A}$, respectively. The latter tangent space is known to be isomorphic to $\Omega^{1}(Y$; ad $\bar{P})$ where ad $\bar{P}=\bar{P} \times_{\text {ad }} \mathfrak{s o}(3)$. A straightforward calculation shows that the curvatures of $A$ and $\bar{A}$ are related by

$$
F_{A}=\pi_{*}^{-1}\left(F_{\bar{A}}\right)+\frac{1}{2} F_{C} \cdot \mathrm{Id} .
$$

We say that $A$ is a projectively flat connection compatible with $C$ if $F_{\bar{A}}=0$. The property of a connection being projectively flat is preserved by the actions of both $\mathcal{G}(P)$ and $H^{1}\left(Y ; \mathbb{Z}_{2}\right)$. The moduli space of projectively flat connections will be denoted by $\mathcal{M}(P)$. 
Fix a projectively flat connection $A_{0}$ compatible with $C$. Define the ChernSimons function $\operatorname{cs}_{A_{0}}: \mathcal{B}(P) \rightarrow \mathbb{R} / \mathbb{Z}$ by the formula

$$
\operatorname{cs}_{A_{0}}(A)=\frac{1}{8 \pi^{2}} \int_{Y} \operatorname{tr}\left(B \wedge d B+\frac{2}{3} B \wedge B \wedge B\right),
$$

where $B=A-A_{0}$. This function is invariant with respect to the $H^{1}\left(Y ; \mathbb{Z}_{2}\right)$-action and defines the Chern-Simons function $\mathbf{c s}_{\bar{A}_{0}}: \mathcal{B}(\bar{P}) \rightarrow \mathbb{R} / 4 \mathbb{Z}$ on the quotient, so that we have the following commutative diagram

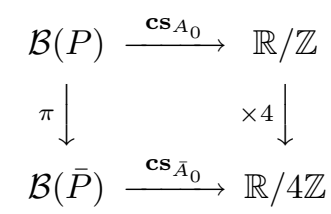

The critical point set of $\mathbf{c s}_{A_{0}}: \mathcal{B}(P) \rightarrow \mathbb{R} / \mathbb{Z}$ can be identified with the moduli space $\mathcal{M}(P)$ of projectively flat connections on $P$, which is independent of the choice of $A_{0}$. For this reason, we will generally omit the $A_{0}$ subscript in what follows. The group $H^{1}\left(Y ; \mathbb{Z}_{2}\right)$ acts on $\mathcal{M}(P)$. The quotient of this action is the flat moduli space $\mathcal{M}(\bar{P})$, which is the critical point set of cs : $\mathcal{B}(\bar{P}) \rightarrow \mathbb{R} / 4 \mathbb{Z}$.

\subsection{Definition of $\lambda^{\prime \prime \prime}$}

Give $Y$ a Riemannian metric, and let $A$ be a projectively flat connection on $P$. The point $[A] \in \mathcal{M}(P)$ is said to be non-degenerate if $H^{1}(Y$; ad $A)=0$. Here, $H^{1}(Y ; \operatorname{ad} A)$ stands for the cohomology with coefficients in the flat bundle ad $\bar{P}$ endowed with the flat connection $\bar{A}$. The moduli space $\mathcal{M}(P)$ is called nondegenerate if all of its points are non-degenerate.

Let $w$ be a non-zero class in $H^{2}\left(Y ; \mathbb{Z}_{2}\right)$, and $P$ a $U(2)$-bundle over $Y$ with $c_{1}(P)=w(\bmod 2)$. If $\mathcal{M}(P)$ is non-degenerate then it is finite and we define the Casson invariant $\lambda^{\prime \prime \prime}(Y, w)$ as

$$
\lambda^{\prime \prime \prime}(Y, w)=\frac{1}{2} \sum_{A \in \mathcal{M}(P)}(-1)^{\mu(A)},
$$

where $\mu(A)$ is the mod 2 Floer index of $A$ defined as in [7, page 150]. Note that the usual Floer index defined modulo 8 is relative; for any pair of projectively flat connections $A_{1}$ and $A_{2}$, the modulo 2 reduction of this relative index equals $\mu\left(A_{1}\right)-\mu\left(A_{2}\right)(\bmod 2)$.

If $\mathcal{M}(P)$ happens to be degenerate then it will need to be perturbed as described in Section 5, and then $\lambda^{\prime \prime \prime}(Y, w)$ will be defined essentially as above. That $\lambda^{\prime \prime \prime}(Y, w)$ is independent of metric and perturbation and is therefore well defined follows from [7, pages 148-149]. 
Proposition 2.2. The action of $H^{1}\left(Y ; \mathbb{Z}_{2}\right)$ preserves the $\bmod 2$ Floer index.

Proof. This follows from [3, pages 239-240].

Remark 2.3. According to Proposition 2.2, the points in the $H^{1}\left(Y ; \mathbb{Z}_{2}\right)$-orbit of a projectively flat connection $A$ are counted in $\lambda^{\prime \prime \prime}(Y, w)$ with the same sign. Hence we could as well define $\lambda^{\prime \prime \prime}(Y, w)$ by counting points in $\mathcal{M}(\bar{P})$, where $w_{2}(\bar{P})=w$, with weights given by the order of the orbits of their respective lifts to $\mathcal{M}(P)$.

\section{Projective representations}

The holonomy map gives a homeomorphism between the moduli space $\mathcal{M}(\bar{P})$ of flat connections on $\bar{P}$ and the $S O(3)$-character variety of $\pi_{1} Y$. Similarly, there is an algebraic interpretation (again using holonomy) of projectively flat connections in terms of projective representations. This section describes this concept in some detail; good general references for these ideas are the classic paper of AtiyahBott [2] and the book of Brown [5].

\subsection{Algebraic background}

Let $G$ be a finitely presented group and view $\mathbb{Z}_{2}=\{ \pm 1\}$ as the center of $S U(2)$. A map $\rho: G \rightarrow S U(2)$ is called a projective representation if $\rho(g h) \rho(h)^{-1} \rho(g)^{-1} \in \mathbb{Z}_{2}$ for all $g, h \in G$. Given a projective representation $\rho$, the function $c: G \times G \rightarrow \mathbb{Z}_{2}$ defined as $c(g, h)=\rho(g h) \rho(h)^{-1} \rho(g)^{-1}$ is a 2-cocycle, that is, $c(g h, k) c(g, h)=$ $c(g, h k) c(h, k)$. We will refer to $c$ as the cocycle associated with $\rho$.

Let us fix a cocycle $c: G \times G \rightarrow \mathbb{Z}_{2}$ and denote by $\mathrm{PR}_{c}(G ; S U(2))$ the set of all projective representations $\rho: G \rightarrow S U(2)$ whose associated 2-cocycle is $c$.

Lemma 3.1. If $c$ and $c^{\prime}: G \times G \rightarrow \mathbb{Z}_{2}$ are cocycles such that $[c]=\left[c^{\prime}\right] \in H^{2}\left(G ; \mathbb{Z}_{2}\right)$ then there is a bijection between $\mathrm{PR}_{c}(G ; S U(2))$ and $\mathrm{PR}_{c^{\prime}}(G ; S U(2))$.

Proof. The fact that $[c]=\left[c^{\prime}\right]$ means that there exists a function $\mu: G \rightarrow \mathbb{Z}_{2}$ such that $\mu(g h) c(g, h)=\mu(g) \mu(h) c^{\prime}(g, h)$ for all $g, h \in G$. Define a map $\varphi$ : $\mathrm{PR}_{c}(G ; S U(2)) \rightarrow \mathrm{PR}_{c^{\prime}}(G ; S U(2))$ by the formula $\varphi(\rho)(g)=\mu(g) \rho(g)$. One can easily check that $\varphi(\rho) \in \mathrm{PR}_{c^{\prime}}(G ; S U(2))$ and that $\varphi$ is a bijection : its inverse $\psi$ : $\mathrm{PR}_{c^{\prime}}(G ; S U(2)) \rightarrow \mathrm{PR}_{c}(G ; S U(2))$ is given by the formula $\psi\left(\rho^{\prime}\right)(g)=\mu(g) \rho^{\prime}(g)$.

Let $c: G \times G \rightarrow \mathbb{Z}_{2}$ be a 2-cocycle and $\rho_{1}, \rho_{2} \in \mathrm{PR}_{c}(G ; S U(2))$. We say that $\rho_{1} \simeq \rho_{2}$ if there exists a function $\mu: G \rightarrow \mathbb{Z}_{2}$ and an element $\sigma \in S U(2)$ such that $\rho_{2}(g)=\mu(g) \sigma \rho_{1}(g) \sigma^{-1}$ for all $g \in G$. 
Lemma 3.2. The map $\mu: G \rightarrow \mathbb{Z}_{2}$ is a homomorphism.

Proof. For any elements $g, h \in G$ we have $\rho_{2}(g h)=c(g, h) \rho_{2}(g) \rho_{2}(h)$. This implies that $\mu(g h) \sigma \rho_{1}(g h) \sigma^{-1}=c(g, h) \mu(g) \sigma \rho_{1}(g) \sigma^{-1} \mu(h) \sigma \rho_{1}(h) \sigma^{-1}$, and, after simplification, $\mu(g h) \rho_{1}(g h)=\mu(g) \mu(h) c(g, h) \rho_{1}(g) \rho_{1}(h)$. Since $\rho_{1}(g h)=c(g, h) \rho_{1}(g) \rho_{1}(h)$, we conclude that $\mu(g h)=\mu(g) \mu(h)$.

Let $\mathcal{P} \mathcal{R}_{c}(G ; S U(2))$ be the set of conjugacy classes of projective representations of $G$ viewed as $S U(2)$ valued functions. One can easily see that $\simeq$ descends to equivalence relation on $\mathcal{P}_{\mathcal{R}}(G ; S U(2))$, and hence there is a natural projection map

$$
\mathcal{P} \mathcal{R}_{c}(G ; S U(2)) \quad \longrightarrow \mathcal{P} \mathcal{R}_{c}(G ; S U(2)) / \simeq \quad=\quad \operatorname{PR}_{c}(G ; S U(2)) / \simeq .
$$

For any choice of cocycle $c: G \times G \rightarrow \mathbb{Z}_{2}$, the set $\operatorname{PR}_{c}(G ; S U(2))$ is acted upon by the group $H^{1}\left(G ; \mathbb{Z}_{2}\right)=\operatorname{Hom}\left(G ; \mathbb{Z}_{2}\right)$. Every $\chi \in \operatorname{Hom}\left(G ; \mathbb{Z}_{2}\right)$ acts by the formula $\rho \mapsto \rho^{\chi}$ where $\rho^{\chi}(g)=\chi(g) \rho(g), g \in G$ (one can easily see that the cocycle associated with $\rho^{\chi}$ is again $c$ ). This action preserves conjugacy, and hence defines an action on $\mathcal{P} \mathcal{R}_{c}(G ; S U(2))$.

Proposition 3.3. The quotient of $\mathcal{P} \mathcal{R}_{c}(G ; S U(2))$ by the $H^{1}\left(G ; \mathbb{Z}_{2}\right)$-action equals $\mathcal{P} \mathcal{R}_{c}(G ; S U(2)) / \simeq$.

Proof. If $\rho_{1} \simeq \rho_{2}$ then there exists a map $\mu: G \rightarrow \mathbb{Z}_{2}$ and an element $\sigma \in S U(2)$ such that $\rho_{2}(g)=\mu(g) \sigma \rho_{1}(g) \sigma^{-1}$. Since $\mu$ is necessarily a homomorphism by Lemma 3.2 , the above equality means that $\rho_{2}$ is conjugate to $\rho_{1}^{\mu}$. The same formula shows that if $\rho_{2}$ is conjugate to $\rho_{1}^{\chi}$ for $\chi \in H^{1}\left(Y ; \mathbb{Z}_{2}\right)$, then $\rho_{1} \simeq \rho_{2}$.

We now want to relate the projective $S U(2)$-representations studied above to the ordinary $S O(3)$-representations of $G$. Let $\alpha: G \rightarrow S O(3)$ be a representation. It yields a map of classifying spaces $B G \rightarrow B S O(3)$. Since $G$ is a discrete group, we can identify $H^{2}\left(B G ; \mathbb{Z}_{2}\right)$ with the group cohomology $H^{2}\left(G ; \mathbb{Z}_{2}\right)$. Thus we obtain a homomorphism

$$
H^{2}\left(B S O(3) ; \mathbb{Z}_{2}\right) \rightarrow H^{2}\left(G ; \mathbb{Z}_{2}\right)
$$

Let $w_{2}(\alpha)$ be the image in $H^{2}\left(G ; \mathbb{Z}_{2}\right)$ of the universal Stiefel-Whitney class $w_{2} \in$ $H^{2}\left(B S O(3) ; \mathbb{Z}_{2}\right)$

Proposition 3.4. Let ad $\rho: G \rightarrow S O(3)$ be the composition of $\rho \in \mathrm{PR}_{c}(G ; S U(2))$ and ad $: S U(2) \rightarrow S O(3)$. Then $\operatorname{ad} \rho$ is a representation, and $w_{2}(\operatorname{ad} \rho)=[c] \in$ $H^{2}\left(G ; \mathbb{Z}_{2}\right)$.

Proof. This follows from the description of $w_{2}(\operatorname{ad} \rho)$ as the obstruction to lifting ad $\rho$ to an $S U(2)$ representation. 
Corollary 3.5. Let $\rho: G \rightarrow S U(2)$ be a projective representation with associated 2-cocycle c. Suppose that there is a non-central element $u \in S U(2)$ such that $u \rho(g)=\rho(g) u$ for all $g \in G$. Then $[c]=0$ in $H^{2}\left(G ; \mathbb{Z}_{2}\right)$.

Proof. The image of $\rho$ is contained in a circle in $S U(2)$ hence ad $\rho$ is conjugate to an $S O(2)$ representation and hence admits an $S U(2)$ lift. This implies that $[c]=w_{2}(\operatorname{ad} \rho)=0$.

A projective representation $\rho: G \rightarrow S U(2)$ is called irreducible if the centralizer of its image equals the center of $S U(2)$. According to the above corollary, any projective representation whose 2-cocycle is not cohomologous to zero is irreducible.

Let $w \in H^{2}\left(G ; \mathbb{Z}_{2}\right)$ and denote by $\mathcal{R}_{w}(G ; S O(3))$ the set of the conjugacy classes of $S O(3)$ representations of $G$ whose second Stiefel-Whitney class equals $w$. This is a compact real algebraic variety. The correspondence $\rho \mapsto \operatorname{ad} \rho$ defines a map

$$
\operatorname{PR}_{c}(G ; S U(2)) / \simeq \longrightarrow \mathcal{R}_{[c]}(G ; S O(3))
$$

Proposition 3.6. The map (4) is a bijection.

Proof. Suppose that $\rho_{1}, \rho_{2} \in \mathrm{PR}_{c}(G ; S U(2))$ are such that ad $\rho_{1}$ and ad $\rho_{2}$ are conjugate as $S O(3)$ representations. Then there exists a function $\mu: G \rightarrow \mathbb{Z}_{2}$ and an element $\sigma \in S U(2)$ such that $\rho_{2}(g)=\mu(g) \sigma \rho_{1}(g) \sigma^{-1}$ for all $g \in G$. This means that $\rho_{1} \simeq \rho_{2}$ and the map (4) is injective.

Given a representation ad $\rho: G \rightarrow S O(3)$, we can always lift it to a projective representation $\rho^{\prime} \in \mathrm{PR}_{c^{\prime}}(G ; S U(2))$ for some $c^{\prime}$ such that $\left[c^{\prime}\right]=[c]$. But then we can also find a lift $\rho \in \mathrm{PR}_{c}(G ; S U(2))$ because $\mathrm{PR}_{c^{\prime}}(G ; S U(2))=\mathrm{PR}_{c}(G ; S U(2))$ by Lemma 3.1 .

In the future, we will simplify the notation $\mathcal{R}_{w}\left(\pi_{1} Y ; S O(3)\right)$ to $\mathcal{R}_{w}(Y ; S O(3))$ etc.

\subsection{The holonomy correspondence}

In this section we establish a correspondence between projectively flat connections over a manifold $Y$ (which is not necessarily a homology 3-torus) and projective representations of its fundamental group. The correspondence is, in rough terms, given by taking the holonomy of a projectively flat connection. In principle, this is well-known, but we could not find a reference. Moreover, some subtle points arise in establishing the continuity of the correspondence.

In what follows, we will use the principle that connections pull back under smooth maps. More precisely, let $j: M \rightarrow W$ be a smooth map and suppose 
that $Q \rightarrow W$ is a principal $G$-bundle with connection, determined by a 1 -form $\omega \in \Omega^{1}(Q ; \mathfrak{g})$, where $\mathfrak{g}$ is the Lie algebra of $G$. There is a bundle map $\bar{j}: j^{*} Q \rightarrow Q$ which commutes with the $G$-actions and which is an isomorphism on the fibers. Then $\bar{j}^{*} \omega$ gives a connection on the bundle $j^{*} Q$, whose holonomy has the following property: If $\gamma: I \rightarrow M$ is a loop, then

$$
\operatorname{hol}_{j^{*} \omega}(\gamma)=\operatorname{hol}_{\omega}\left(j_{*} \gamma\right)
$$

Having said that, consider the natural map $Y \rightarrow B\left(\pi_{1} Y\right)$. It induces a monomorphism $\iota: H^{2}\left(\pi_{1} Y ; \mathbb{Z}_{2}\right) \rightarrow H^{2}\left(Y ; \mathbb{Z}_{2}\right)$, see [5]. We first deal with the discrepancy arising from the fact that $\iota$ need not be surjective.

Lemma 3.7. Let $P$ be a $U(2)$-bundle over a manifold $Y$ such that $w_{2}(\bar{P})$ is not in the image of $\iota: H^{2}\left(\pi_{1} Y ; \mathbb{Z}_{2}\right) \rightarrow H^{2}\left(Y ; \mathbb{Z}_{2}\right)$. Then the moduli space $\mathcal{M}(P)$ is empty.

Proof. The Hopf exact sequence $\pi_{2} Y \rightarrow H_{2}(Y ; \mathbb{Z}) \rightarrow H_{2}\left(\pi_{1} Y ; \mathbb{Z}\right) \rightarrow 0$, see [5], implies that, if $w_{2}(\bar{P})$ does not belong to the image of $\iota$, it evaluates non-trivially on a 2 -sphere in $Y$. Such a bundle $\bar{P}$ cannot support any flat connections, for a flat connection on $\bar{P}$ would pull back to a flat connection on the 2 -sphere, whose holonomy would trivialize the bundle.

For the rest of this subsection, we will concentrate on bundles $P$ such that $w_{2}(\bar{P})$ is in the image of $H^{2}\left(\pi_{1} Y ; \mathbb{Z}_{2}\right)$, and will identify $H^{2}\left(\pi_{1} Y ; \mathbb{Z}_{2}\right)$ with its (monomorphic) image in $H^{2}\left(Y ; \mathbb{Z}_{2}\right)$.

It is a well known fact that the holonomy defines a bijection $\bar{\varphi}: \mathcal{M}(\bar{P}) \rightarrow$ $\mathcal{R}_{w}(Y ; S O(3))$ where $w_{2}(\bar{P})=w(\bmod 2)$. Given a $U(2)$-bundle $P$ with $c_{1}(P)=$ $w(\bmod 2)$, our immediate goal will be to define an $H^{1}\left(Y ; \mathbb{Z}_{2}\right)$-equivariant map $\varphi: \mathcal{M}(P) \rightarrow \mathcal{P} \mathcal{R}_{c}(Y ; S U(2))$, where $[c]=w$, which makes the following diagram commute

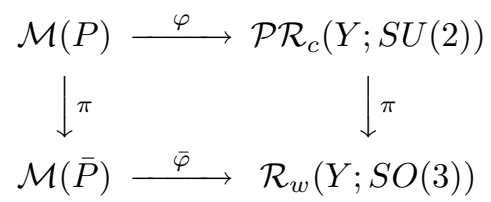

Here, $\pi: \mathcal{P} \mathcal{R}_{c}(Y ; S U(2)) \rightarrow \mathcal{R}_{w}(Y ; S O(3))$ is the map (3) followed by the bijection (4), see Section 3. It is straightforward to define a map $A \rightarrow \varphi(A)$ by lifting $\bar{\varphi}(\bar{A})$ to a projective representation. However, such an assignment might not be continuous, because the choice of lifting is not canonical.

Let $A$ be a projectively flat connection on $P$ whose central part is a fixed connection $C$ on the line bundle $\operatorname{det} P$. For any based loop $\gamma$ in $Y$, we let

$$
\varphi(A)(\gamma)=\operatorname{hol}_{A}(\gamma) \cdot \operatorname{hol}_{C}(\gamma)^{-1 / 2} \in S U(2)
$$

where the square root of $\operatorname{hol}_{C}(\gamma) \in U(1)$ in the second factor is defined as follows. Let $\Omega(Y)$ be the monoid of based loops in $Y$, and fix a representative $\gamma$ in each 
connected component $\Omega_{[\gamma]}$ so that

$$
\Omega(Y)=\bigsqcup_{[\gamma] \in \pi_{1} Y} \Omega_{[\gamma]}
$$

Choose a square root $\operatorname{hol}_{C}(\gamma)^{1 / 2} \in U(1)$ for each of the $\gamma$ and define a (based) map

$$
h:\left(\Omega_{[\gamma]}, \gamma\right) \rightarrow(U(1), 1)
$$

by the formula $h(\alpha)=\operatorname{hol}_{C}(\alpha) \cdot \operatorname{hol}_{C}(\gamma)^{-1}$. If $\pi: \underset{\sim}{U}(1) \rightarrow U(1)$ is the squaring map then we want to lift $h$ to $\tilde{h}$ such that $h=\pi \circ \tilde{h}$ (given such a lift, we get a square root of $\operatorname{hol}_{C}(\alpha)$ by the formula $\left.\tilde{h}(\alpha) \cdot \operatorname{hol}_{C}(\gamma)^{1 / 2}\right)$. The obstruction to the above lifting problem is given by

$$
\begin{aligned}
\mathcal{O} & \in H^{1}\left(\Omega_{[\gamma]}, \gamma ; \mathbb{Z}_{2}\right) \\
& =\operatorname{Hom}\left(H_{1}\left(\Omega_{[\gamma]}, \gamma ; \mathbb{Z}\right) ; \mathbb{Z}_{2}\right) \\
& =\operatorname{Hom}\left(\pi_{1}\left(\Omega_{[\gamma]}, \gamma\right) ; \mathbb{Z}_{2}\right) \\
& =\operatorname{Hom}\left(\pi_{1}\left(\Omega_{*}, *\right) ; \mathbb{Z}_{2}\right), \quad \text { where } * \text { is the trivial loop, } \\
& =\operatorname{Hom}\left(\pi_{2}(Y, *) ; \mathbb{Z}_{2}\right) .
\end{aligned}
$$

In particular, we immediately see that this obstruction vanishes as long as $\pi_{2}(Y)=0$.

Lemma 3.8. For any $U(2)$-bundle $P$ such that $c_{1}(P)=w \neq 0(\bmod 2)$, the obstruction $\mathcal{O}$ is zero.

Proof. Without loss of generality we may assume that $\gamma=*$, the trivial loop. The obstruction $\mathcal{O}$ can be described as follows. Given a homotopy $\alpha_{t}, 0 \leq t \leq 1$, such that $\alpha_{0}=\alpha_{1}=*$ define $\tilde{h}\left(\alpha_{0}\right)=1$, and $\tilde{h}\left(\alpha_{t}\right)$ by path lifting. Then $\mathcal{O}(\sigma)=$ $\tilde{h}\left(\alpha_{1}\right) \in \mathbb{Z}_{2}$. The image of $\mathcal{O}$ in $\operatorname{Hom}\left(\pi_{2}(Y, *) ; \mathbb{Z}_{2}\right)$ is gotten by viewing a 2 -sphere $\sigma$ in $Y$ as such a path.

Now, given a class $\sigma \in \pi_{2}(Y)$, the obstruction to extracting a root of the bundle $\operatorname{det} P$ is given by evaluation of $\sigma^{*}\left(w_{2}(\operatorname{det} P)\right)$ on $S^{2}$. Since $w_{2}(\operatorname{det} P)=w_{2}(\bar{P})$ and $\bar{P}$ admits a flat connection, the latter evaluation has to be zero. Thus the bundle $\operatorname{det} P$ admits a square root over every 2 -sphere, and the holonomy of this square root along the loops $\alpha_{t}$ gives a lift $\tilde{h}$ which necessarily satisfies $\tilde{h}\left(\alpha_{0}\right)=\tilde{h}\left(\alpha_{1}\right)$. In particular, $\mathcal{O}$ vanishes.

With the above definition of $\operatorname{hol}_{C}(\gamma)^{1 / 2}$ in place, the map $\varphi$ is defined by the formula (5). For any projectively flat connection $A$ in $P$, composition of $\varphi(A)$ with the natural projection $S U(2) \rightarrow S O(3)$ gives a representation $\bar{\varphi}(\bar{A}): \pi_{1} Y \rightarrow$ $S O(3)$. In particular, if $\left[\gamma_{1}\right]=\left[\gamma_{2}\right]$ then $\varphi(A)\left(\gamma_{1}\right)= \pm \varphi(A)\left(\gamma_{2}\right)$. Together with continuity of $\varphi(A)$ this implies that $\varphi(A)\left(\gamma_{1}\right)=\varphi(A)\left(\gamma_{2}\right)$ and hence $\varphi(A): \pi_{1} Y \rightarrow$ $S U(2)$ is a well defined projective representation. 
Proposition 3.9. Let $P$ be a $U(2)$-bundle over $Y$ such that $c_{1}(P)=w \neq 0$ $(\bmod 2)$. Then $\varphi$ is well defined as a map $\varphi: \mathcal{M}(P) \rightarrow \mathcal{P R}_{c}(Y ; S U(2))$ with $[c]=w$, and it is an $H^{1}\left(Y ; \mathbb{Z}_{2}\right)$-equivariant bijection.

Proof. The connection $C$ on $\operatorname{det} P$ does not change when $A$ is replaced by a gauge equivalent connection. Therefore, the second factor in (5) remains unchanged and the first one changes by conjugation. Since $\operatorname{hol}_{C}(\gamma)^{-1 / 2}$ is central, the entire $\varphi(A)$ changes by conjugation. Therefore, the map $\varphi: \mathcal{M}(P) \rightarrow \mathcal{P} \mathcal{R}_{c}(Y ; S U(2))$ is well defined. The cocycle $c$ is determined by the choice of $\operatorname{hol}_{C}(\gamma)^{1 / 2}$ for the representative loops $\gamma$, different choices leading to cohomologous cocycles. That $[c]=w$ can be read off the definition of $\varphi$.

Let $\chi \in H^{1}\left(Y ; \mathbb{Z}_{2}\right)$ and replace $A$ by $A \otimes \chi$ then $A$ and $A \otimes \chi$ induce the same connection $C$ on $\operatorname{det} P$ so that the second factor in (5) stays the same. The first factor becomes $\operatorname{hol}_{A \otimes \chi}(\gamma)=\chi(\gamma) \cdot \operatorname{hol}_{A}(\gamma)$ with $\chi(\gamma)= \pm 1$. Hence $\varphi$ is $H^{1}\left(Y ; \mathbb{Z}_{2}\right)$-equivariant. Since its quotient map $\bar{\varphi}$ is a bijection, so is $\varphi$.

An argument similar to that for representation varieties shows that Zariski tangent space to $\mathcal{P} \mathcal{R}_{c}(Y ; S U(2))$ at a projective representation $\rho: \pi_{1} Y \rightarrow S U(2)$ equals $H^{1}(Y$; ad $\rho)$ where ad $\rho: \pi_{1} Y \rightarrow S U(2) \rightarrow S O(3)$ is a representation. It is identified as usual with the tangent space to $\mathcal{M}(P)$ at the corresponding projectively flat connection.

\subsection{Application to homology 3-tori}

A homology torus $Y$ is called odd if there exist vectors $a_{1}, a_{2}$, and $a_{3}$ in $H^{1}\left(Y ; \mathbb{Z}_{2}\right)$ such that $\left(a_{1} \cup a_{2} \cup a_{3}\right)[Y]=1(\bmod 2)$. Note that such $a_{1}, a_{2}$, and $a_{3}$ form a basis of $H^{1}\left(Y ; \mathbb{Z}_{2}\right)$ because they are distinguished by cup-products with $a_{1} \cup a_{2}, a_{2} \cup a_{3}$, and $a_{1} \cup a_{3}$ and hence are linearly independent. Also note that if $\left(a_{1} \cup a_{2} \cup a_{3}\right)[Y]=$ $1(\bmod 2)$ for some basis $a_{1}, a_{2}, a_{3} \in H^{1}\left(Y ; \mathbb{Z}_{2}\right)$ then the same is true for any other basis. A homology torus $Y$ is called even if $\left(a_{1} \cup a_{2} \cup a_{3}\right)[Y]=0(\bmod 2)$ for any three vectors $a_{1}, a_{2}, a_{3} \in H^{1}\left(Y ; \mathbb{Z}_{2}\right)$.

Let $\Lambda^{2} H^{1}\left(Y ; \mathbb{Z}_{2}\right)$ be the second exterior power of $H^{1}\left(Y ; \mathbb{Z}_{2}\right)$ and consider the cup-product map

$$
\cup: \Lambda^{2} H^{1}\left(Y ; \mathbb{Z}_{2}\right) \rightarrow H^{2}\left(Y ; \mathbb{Z}_{2}\right)
$$

Lemma 3.10. The map (6) is an isomorphism if $Y$ is odd, and is zero if $Y$ is even.

Proof. Let $Y$ be an odd homology torus and choose a basis $a_{1}, a_{2}, a_{3} \in H^{1}\left(Y ; \mathbb{Z}_{2}\right)$. The vectors $a_{1} \cup a_{2}, a_{2} \cup a_{3}$, and $a_{1} \cup a_{3} \in H^{2}\left(Y ; \mathbb{Z}_{2}\right)$ are linearly independent because they are distinguished by the homomorphisms $H^{2}\left(Y ; \mathbb{Z}_{2}\right) \rightarrow H^{3}\left(Y ; \mathbb{Z}_{2}\right)$ given by cup-products with $a_{1}, a_{2}$, and $a_{3}$. Hence (6) is an isomorphism. 
Suppose now that $Y$ is even and that there are vectors $a, b \in H^{1}\left(Y ; \mathbb{Z}_{2}\right)$ such that $a \cup b \neq 0(\bmod 2)$. By Poincaré duality, there exists $c \in H^{1}\left(Y ; \mathbb{Z}_{2}\right)$ such that $a \cup b \cup c=1(\bmod 2)$, a contradiction.

Corollary 3.11. If $Y$ is odd then the map $\iota: H^{2}\left(\pi_{1} Y ; \mathbb{Z}_{2}\right) \rightarrow H^{2}\left(Y ; \mathbb{Z}_{2}\right)$ is an isomorphism.

Proof. This follows from the commutative diagram

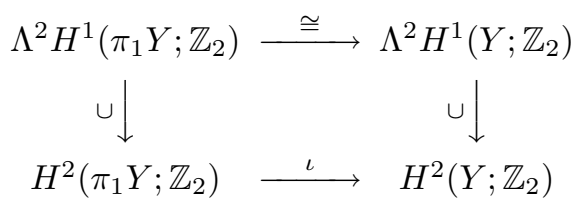

whose upper arrow is an isomorphism because $H^{1}\left(\pi_{1} Y ; \mathbb{Z}_{2}\right)=H^{1}\left(Y ; \mathbb{Z}_{2}\right)$, and whose right arrow is an isomorphism by Lemma 3.10. Since $\iota$ is injective, the remaining two arrows in the diagram are also isomorphisms.

Note that if $Y$ is an even homology torus, the conclusion of Corollary 3.11 need no longer hold: take for example $Y=\left(S^{1} \times S^{2}\right) \#\left(S^{1} \times S^{2}\right) \#\left(S^{1} \times S^{2}\right)$.

Corollary 3.12. Theorem 1.1 holds for all $\lambda^{\prime \prime \prime}(Y, w)$ such that $w$ is not in the image of $\iota: H^{2}\left(\pi_{1} Y ; \mathbb{Z}_{2}\right) \rightarrow H^{2}\left(Y ; \mathbb{Z}_{2}\right)$.

Proof. Let $P$ be a bundle with $w_{2}(\bar{P})=w$ not in the image of $\iota$. Then, according to Lemma 3.7, the moduli space $\mathcal{M}(P)$ is empty so that $\lambda^{\prime \prime \prime}(Y, w)=0$. On the other hand, this situation is only possible if $Y$ is an even homology torus, see Corollary 3.11 .

We will assume from now on that $w=w_{2}(\bar{P})$ is in the image of $\iota$ and will not make distinction between $H^{2}\left(\pi_{1} Y ; \mathbb{Z}_{2}\right)$ and its (monomorphic) image in $H^{2}\left(Y ; \mathbb{Z}_{2}\right)$. Because of the identification of Proposition 3.9, the Casson invariant $\lambda^{\prime \prime \prime}(Y, w)$ can be defined by counting points in the space $\mathcal{P R}_{c}(Y ; S U(2))$ with $[c]=w$, perhaps after perturbation.

\section{The two-orbits}

According to the action of $H^{1}\left(Y ; \mathbb{Z}_{2}\right)=\left(\mathbb{Z}_{2}\right)^{3}$ the space $\mathcal{P} \mathcal{R}_{c}(Y ; S U(2))$ splits into orbits of possible orders one, two, four, and eight. In this section we study the two-orbits (orbits with two elements, or those with stabilizer $\mathbb{Z}_{2} \oplus \mathbb{Z}_{2}$ ). 


\subsection{The two-orbits and invariant $\lambda^{\prime \prime \prime}$}

Consider a subgroup of $S O(3)$ that is isomorphic to $\mathbb{Z}_{2} \oplus \mathbb{Z}_{2}$. Such a subgroup is generated by $180^{\circ}$ rotations about two perpendicular axes in $\mathbb{R}^{3}$, and any two such subgroups are conjugate to each other in $S O(3)$. Hence the following definition makes sense. Define $\mathcal{R}_{w}\left(Y ; \mathbb{Z}_{2} \oplus \mathbb{Z}_{2}\right)$ to be the subspace of $\mathcal{R}_{w}(Y ; S O(3))$ consisting of the $S O(3)$ conjugacy classes of representations $\alpha: \pi_{1} Y \rightarrow S O(3)$ which factor through $\mathbb{Z}_{2} \oplus \mathbb{Z}_{2} \subset S O(3)$.

Proposition 4.1. Let $[c]=w$ be a non-trivial class in $H^{2}\left(Y ; \mathbb{Z}_{2}\right)$. Then the map $\pi: \mathcal{P}_{\mathcal{R}}(Y ; S U(2)) \rightarrow \mathcal{R}_{w}(Y ; S O(3))$ establishes a bijective correspondence between the set of two-orbits in $\mathcal{P} \mathcal{R}_{c}(Y ; S U(2))$ and the set $\mathcal{R}_{w}\left(Y ; \mathbb{Z}_{2} \oplus \mathbb{Z}_{2}\right)$.

Proof. Suppose that the conjugacy class of a projective representation $\rho: \pi_{1} Y \rightarrow$ $S U(2)$ is fixed by a subgroup $\mathbb{Z}_{2} \oplus \mathbb{Z}_{2}$ of $H^{1}\left(Y ; \mathbb{Z}_{2}\right)$ generated by homomorphisms $\alpha, \beta: \pi_{1} Y \rightarrow \mathbb{Z}_{2}$. Then there exists a $u \in S U(2)$ such that $\alpha(x) \rho(x)=u \rho(x) u^{-1}$ for all $x \in \pi_{1} Y$. Observe that $\rho(x)=u^{2} \rho(x) u^{-2}$ and, since $\rho$ is irreducible, $u^{2}= \pm 1$. The case $u^{2}=1$ should be excluded because $u^{2}=1$ would imply that $u= \pm 1$ so that $-\rho(x)=\rho(x)$ at least for some $x$, which is impossible in $S U(2)$. Therefore $u^{2}=-1$ and, after conjugation if necessary, we may assume that $u=i$. Then, for every $x \in \pi_{1} Y$, we have $\pm \rho(x)=i \rho(x) i^{-1}$ so that $\operatorname{im}(\rho) \subset S_{i} \cup j \cdot S_{i}$. Here, $S_{i}$ is the complex circle in $S U(2)$ (and $S U(2)$ is viewed as the group of unit quaternions).

Similarly, there exists a $v \in S U(2)$ such that $\beta(x) \rho(x)=v \rho(x) v^{-1}$ and $v^{2}=$ -1 . After conjugation by a complex number, we may assume that $v=i a+b j$ where $a, b \in \mathbb{R}$ and $b \geq 0$. Next, $\alpha(x) \beta(x) \rho(x)=(i v) \rho(x)(i v)^{-1}$ so that $(i v)^{2}=-1$. An easy calculation with quaternions shows that $v=j$ (and then $i v=k$ ). Thus $\rho$ has the property that $\pm \rho(x)=i \rho(x) i^{-1}$ and $\pm \rho(x)=j \rho(x) j^{-1}$ for all $x \in \pi_{1} Y$. Therefore,

$$
\operatorname{im}(\rho) \subset\left(S_{i} \cup j \cdot S_{i}\right) \cap\left(S_{j} \cup i \cdot S_{j}\right)
$$

where $S_{j}$ is the circle of quaternions of the form $\exp (j \varphi)$. One can easily see that the latter intersection is the group $Q=\{ \pm 1, \pm i, \pm j, \pm k\}$.

The above argument shows that any projective representation $\rho: \pi_{1} Y \rightarrow S U(2)$ stabilized by $\mathbb{Z}_{2} \oplus \mathbb{Z}_{2}$ factors through $Q$ and therefore its associated $S O(3)$-representation ad $\rho$ factors through a copy of $\mathbb{Z}_{2} \oplus \mathbb{Z}_{2} \subset S O(3)$.

To complete the proof, we only need to show that the orbit of $\rho$ consists of exactly two points. Let $\gamma$ be a vector in $H^{1}\left(Y ; \mathbb{Z}_{2}\right)$ completing $\alpha, \beta$ to a basis. Then $\rho$ and $\rho^{\gamma}$ lie in the same $H^{1}\left(Y ; \mathbb{Z}_{2}\right)$-orbit but are not conjugate. The latter can be seen as follows: if there exists a $w \in S U(2)$ such that $\gamma(x) \rho(x)=w \rho(x) w^{-1}$ then $w= \pm k$ and $\alpha(x) \beta(x) \gamma(x) \rho(x)=(i j k) \rho(x)(i j k)^{-1}=\rho(x)$ for all $x$, a contradiction.

Remark 4.2. The above proof shows in particular that no point of $\mathcal{P} \mathcal{R}_{c}(Y ; S U(2))$ 
with $[c] \neq 0$ is fixed by the entire group $H^{1}\left(Y ; \mathbb{Z}_{2}\right)$ so that $\mathcal{P} \mathcal{R}_{c}(Y ; S U(2))$ has no orbits of order one.

\subsection{The number of two-orbits}

Our next goal is to find a formula for the number of points in $\mathcal{R}_{w}\left(Y ; \mathbb{Z}_{2} \oplus \mathbb{Z}_{2}\right)$ modulo 2 .

Proposition 4.3. Let $0 \neq w \in H^{2}\left(Y ; \mathbb{Z}_{2}\right)$ then $\# \mathcal{R}_{w}\left(Y ; \mathbb{Z}_{2} \oplus \mathbb{Z}_{2}\right)=\left(a_{1} \cup a_{2} \cup\right.$ $\left.a_{3}\right)[Y](\bmod 2)$. Moreover, if $Y$ is even then $\mathcal{R}_{w}\left(Y ; \mathbb{Z}_{2} \oplus \mathbb{Z}_{2}\right)$ is empty.

Proof. We begin by observing that any two subgroups of $S O(3)$ that are isomorphic to $\mathbb{Z}_{2} \oplus \mathbb{Z}_{2}$ are conjugate, and that moreover any automorphism of such a subgroup is realized by conjugation by an element of $S O(3)$. Let us fix a subgroup $\mathbb{Z}_{2} \oplus \mathbb{Z}_{2}$ and a basis in it.

Since $\mathbb{Z}_{2} \oplus \mathbb{Z}_{2}$ is abelian, every $\alpha \in \mathcal{R}_{w}\left(Y ; \mathbb{Z}_{2} \oplus \mathbb{Z}_{2}\right)$ factors through a homomorphism $H_{1}(Y ; \mathbb{Z}) \rightarrow \mathbb{Z}_{2} \oplus \mathbb{Z}_{2}$. The two components of this homomorphism determine elements $\beta, \gamma \in \operatorname{Hom}\left(H_{1}(Y ; \mathbb{Z}) ; \mathbb{Z}_{2}\right) \cong H^{1}\left(Y ; \mathbb{Z}_{2}\right)$. It is straightforward to see that the $S O(3)$-representation $\alpha$ may be recovered from $\beta$ and $\gamma$ via the formula $\alpha \cong \beta \oplus \gamma \oplus \operatorname{det}(\beta \oplus \gamma)$. Since any element of $\Lambda^{2} H^{1}\left(Y ; \mathbb{Z}_{2}\right)$ can be represented in the form $\beta \wedge \gamma$, this establishes a one-to-one correspondence

$$
\mathcal{R}\left(Y ; \mathbb{Z}_{2} \oplus \mathbb{Z}_{2}\right) \rightarrow \Lambda^{2} H^{1}\left(Y ; \mathbb{Z}_{2}\right),
$$

where $\mathcal{R}\left(Y ; \mathbb{Z}_{2} \oplus \mathbb{Z}_{2}\right)$ is union of $\mathcal{R}_{w}\left(Y ; \mathbb{Z}_{2} \oplus \mathbb{Z}_{2}\right)$ over all possible $w$. Since $H_{1}(Y ; \mathbb{Z})$ is torsion free, any element in $H^{1}\left(Y ; \mathbb{Z}_{2}\right)$ is the mod 2 reduction of a class in $H^{1}(Y ; \mathbb{Z})$. It follows that the cup product of any element $a \in H^{1}\left(Y ; \mathbb{Z}_{2}\right)$ with itself is 0 . We compute

$$
\begin{aligned}
w_{2}(\alpha) & =w_{1}(\beta) \cup w_{1}(\gamma)+w_{1}(\beta) \cup w_{1}(\operatorname{det}(\beta \oplus \gamma))+w_{1}(\gamma) \cup w_{1}(\operatorname{det}(\beta \oplus \gamma)) \\
& =w_{1}(\beta) \cup w_{1}(\gamma)+w_{1}(\beta) \cup\left(w_{1}(\beta)+w_{1}(\gamma)\right)+w_{1}(\gamma) \cup\left(w_{1}(\beta)+w_{1}(\gamma)\right) \\
& =w_{1}(\beta) \cup w_{1}(\gamma) .
\end{aligned}
$$

Since $w_{1}(\beta)=\beta$ and $w_{1}(\gamma)=\gamma$, this shows that $w_{2}(\alpha)$ is the image of $\beta \wedge \gamma$ under the map (6).

The result now follows by composing (6) and (7): if the triple cup product on $H^{1}\left(Y ; \mathbb{Z}_{2}\right)$ vanishes mod 2 then the map (6) is identically zero, hence $\mathcal{R}_{w}\left(Y ; \mathbb{Z}_{2} \oplus\right.$ $\mathbb{Z}_{2}$ ) is empty for $w \neq 0$. If the triple cup product is nontrivial mod 2 then the map (6) is an isomorphism and $\mathcal{R}_{w}\left(Y ; \mathbb{Z}_{2} \oplus \mathbb{Z}_{2}\right)$ consists of exactly one element for every choice of $0 \neq w \in H^{2}\left(Y ; \mathbb{Z}_{2}\right)$. 


\subsection{Non-degeneracy of the two-orbits}

We wish to use Proposition 4.3 to calculate the contribution of the two-orbits to $\lambda^{\prime \prime \prime}(Y, w)(\bmod 2)$. In order to do that, we need to check the non-degeneracy condition for such orbits in the case when the triple cup product on $Y$ is non-trivial.

Proposition 4.4. Let $Y$ be an odd homology torus then $H^{1}(Y ; \operatorname{ad} \rho)=0$ for any projective representation $\rho: \pi_{1} Y \rightarrow S U(2)$ such that ad $\rho \in \mathcal{R}_{w}\left(Y ; \mathbb{Z}_{2} \oplus \mathbb{Z}_{2}\right)$ with $w \neq 0$.

Notice that any ad $\rho \in \mathcal{R}_{w}\left(Y ; \mathbb{Z}_{2} \oplus \mathbb{Z}_{2}\right)$ splits as ad $\rho=\alpha_{1} \oplus \alpha_{2} \oplus \alpha_{3}$ where each $\alpha_{i}: \pi_{1} Y \rightarrow \mathbb{Z}_{2}$ is a non-trivial representation, and

$$
H^{1}(Y ; \operatorname{ad} \rho)=H^{1}\left(Y ; \alpha_{1}\right) \oplus H^{1}\left(Y ; \alpha_{2}\right) \oplus H^{1}\left(Y ; \alpha_{3}\right),
$$

compare with the proof of Proposition 4.3. Therefore, to prove Proposition 4.4, it is sufficient to show that $H^{1}(Y ; \alpha)$ vanishes for all non-trivial representations $\alpha: \pi_{1} Y \rightarrow \mathbb{Z}_{2}$. Given such a non-trivial representation, let $\pi: Y_{\alpha} \rightarrow Y$ be the regular double covering of $Y$ with $\pi_{1}\left(Y_{\alpha}\right)=\operatorname{ker}(\alpha)$.

Lemma 4.5. The group $H^{1}(Y ; \alpha)$ is isomorphic to the $(-1)$-eigenspace of $\mathbb{Z}_{2}$ acting on $H^{1}\left(Y_{\alpha} ; \mathbb{R}\right)$.

Proof. This is immediate from the definition of $H^{1}(Y ; \alpha)=H^{1}\left(Y ; \mathbb{R}_{\alpha}\right)$ after we identify $\mathbb{Z}_{2}$ with $O(1)$.

Lemma 4.6. The cup product map $\cup a: H^{1}\left(Y ; \mathbb{Z}_{2}\right) \rightarrow H^{2}\left(Y ; \mathbb{Z}_{2}\right)$ is non-trivial for some $a \in H^{1}\left(Y ; \mathbb{Z}_{2}\right)$ if and only if $Y$ is an odd homology torus.

Proof. Suppose that $a \cup b \neq 0$. By Poincaré duality, there is $c \in H^{1}\left(Y ; \mathbb{Z}_{2}\right)$ with $a \cup b \cup c \neq 0$. Conversely, note that the cup product of any three basis elements is the same as the cup product of any other three basis elements. So if there is a non-zero cup product, extend $\{a\}$ to a basis $\{a, b, c\}$ with $a \cup b \cup c \neq 0$. In particular $a \cup b \neq 0$ (and also $a \cup c \neq 0$.)

Remark 4.7. Note that we in fact proved that, if $Y$ is an odd homology torus, the rank of $\cup a: H^{1}\left(Y ; \mathbb{Z}_{2}\right) \rightarrow H^{2}\left(Y ; \mathbb{Z}_{2}\right)$ equals two for any nonzero $a \in H^{1}\left(Y ; \mathbb{Z}_{2}\right)$.

Lemma 4.8. If $Y$ is an odd homology torus then $H^{1}\left(Y_{\alpha} ; \mathbb{Z}\right)=\mathbb{Z}^{3}$.

Proof. Let us consider the Gysin exact sequence for the double covering $\pi: Y_{\alpha} \rightarrow Y$ 
(with coefficients in $\mathbb{Z}_{2}$ )

$$
\begin{aligned}
H^{0}(Y) \stackrel{\alpha}{\rightarrow} H^{1}(Y) \stackrel{\pi^{*}}{\longrightarrow} & H^{1}\left(Y_{\alpha}\right) \\
& \longrightarrow H^{1}(Y) \stackrel{\alpha}{\longrightarrow} H^{2}(Y) \stackrel{\pi^{*}}{\longrightarrow} H^{2}\left(Y_{\alpha}\right) \rightarrow H^{2}(Y) \stackrel{\alpha}{\rightarrow} H^{3}(Y)
\end{aligned}
$$

where the arrows marked $\alpha$ stand for the homomorphisms given by the cup product with $w_{1}(\alpha) \in H^{1}\left(Y ; \mathbb{Z}_{2}\right)$. This sequence works out to

$$
\begin{aligned}
0 \rightarrow \mathbb{Z}_{2} \stackrel{\alpha}{\longrightarrow}\left(\mathbb{Z}_{2}\right)^{3} \stackrel{\pi^{*}}{\longrightarrow} H^{1}\left(Y_{\alpha}\right) & \\
\longrightarrow & \left(\mathbb{Z}_{2}\right)^{3} \stackrel{\alpha}{\longrightarrow}\left(\mathbb{Z}_{2}\right)^{3} \stackrel{\pi^{*}}{\longrightarrow} H^{2}\left(Y_{\alpha}\right) \rightarrow\left(\mathbb{Z}_{2}\right)^{3} \stackrel{\alpha}{\longrightarrow} \mathbb{Z}_{2} \rightarrow 0
\end{aligned}
$$

The first and the last zeroes are because $w_{1}(\alpha) \neq 0$. According to Remark 4.7, the image of $\cup w_{1}(\alpha):\left(\mathbb{Z}_{2}\right)^{3} \rightarrow\left(\mathbb{Z}_{2}\right)^{3}$ has rank two. Counting ranks we get that $H^{1}\left(Y_{\alpha} ; \mathbb{Z}_{2}\right)=H^{2}\left(Y_{\alpha} ; \mathbb{Z}_{2}\right)=\left(\mathbb{Z}_{2}\right)^{3}$. The result now follows by the universal coefficient theorem.

Proof of Proposition 4.4. Let us fix an isomorphism between integral homology of $Y$ and that of the 3 -torus $T$, and choose a map $f: Y \rightarrow T$ that induces this isomorphism. Let $T_{\alpha}$ be the double covering of $T$ corresponding to the homomorphism $\alpha: H_{1}(T ; \mathbb{Z}) \rightarrow \mathbb{Z}_{2}$ which makes the following diagram commute

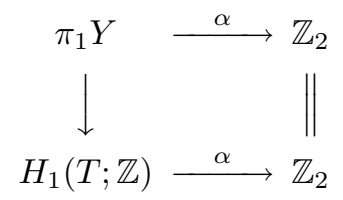

The map $\pi_{1} Y \rightarrow H_{1}(T ; \mathbb{Z})$ in this diagram is obtained by composing the abelianization $\pi_{1} Y \rightarrow H_{1}(Y ; \mathbb{Z})$ with the isomorphism $f_{*}: H_{1}(Y ; \mathbb{Z}) \rightarrow H_{1}(T ; \mathbb{Z})$.

Let $f_{\alpha}: Y_{\alpha} \rightarrow T_{\alpha}$ be a lift of $f$. Comparing Gysin exact sequences of $\pi: Y_{\alpha} \rightarrow Y$ and $\pi: T_{\alpha} \rightarrow T$ using the five-lemma, we conclude that the map $f_{\alpha}^{*}: H^{1}\left(T_{\alpha} ; \mathbb{Z}\right) \rightarrow H^{1}\left(Y_{\alpha} ; \mathbb{Z}\right)$ is an isomorphism when tensored with $\mathbb{Z}_{2}$. Since $H^{1}\left(Y_{\alpha} ; \mathbb{Z}\right)=\mathbb{Z}^{3}$ by Lemma 4.8 , we also conclude that $f_{\alpha}^{*}: H^{1}\left(T_{\alpha} ; \mathbb{R}\right) \rightarrow H^{1}\left(Y_{\alpha} ; \mathbb{R}\right)$ is an isomorphism.

This implies that $\pi^{*}: H^{1}(Y ; \mathbb{R}) \rightarrow H^{1}\left(Y_{\alpha} ; \mathbb{R}\right)$ is an isomorphism, for this is true for $Y=T$, and we just observed the isomorphism in the upper line of the following commutative diagram

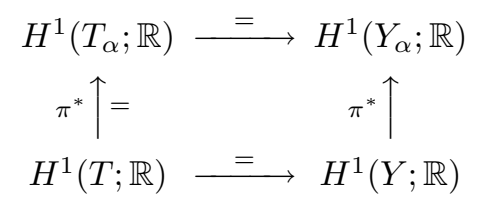

On the other hand, the image of $\pi^{*}: H^{1}(Y ; \mathbb{R}) \rightarrow H^{1}\left(Y_{\alpha} ; \mathbb{R}\right)$ equals the $(+1)$ eigenspace of $\mathbb{Z}_{2}$ acting on $H^{1}\left(Y_{\alpha} ; \mathbb{R}\right)$. Together with Lemma 4.5 , this implies that $H^{1}(Y ; \alpha)=H^{1}\left(Y ; \mathbb{R}_{\alpha}\right)=0$. 


\section{Perturbations}

In this section we deal with the situation when the critical point set $\mathcal{M}(P)$ of cs $: \mathcal{B}(P) \rightarrow \mathbb{R} / \mathbb{Z}$ is degenerate. We describe a class of equivariant admissible perturbations $h: \mathcal{B}(P) \rightarrow \mathbb{R}$ and prove that, for a small generic $h$, the critical point set of $\mathbf{c s}+h$ is non-degenerate. This set is acted upon by $H^{1}\left(Y ; \mathbb{Z}_{2}\right)$ in such a manner that an argument similar to that used in the non-degenerate case completes the proof of Theorem 1.1, see Section 6 .

We define our perturbations using holonomy around loops, following the approach originated by Taubes, Floer and Donaldson [20, 8, 7]. While realizing this approach in the equivariant setting, we have to address certain new issues. First, we need to make the perturbations $H^{1}\left(Y ; \mathbb{Z}_{2}\right)$-equivariant; this is done by imposing a simple homological restriction on the loops. Next, we need to show that these equivariant perturbations are generic; in addition to the usual perturbation theory, this requires more refined 'second order' perturbation arguments. The latter are described in Herald [10] and in [11], compare with Section 5.6.

\subsection{Equivariant admissible perturbations}

Let $\gamma_{k}: S^{1} \rightarrow Y, k=1, \ldots, n$, be a collection of closed embedded loops in $Y$ which are disjoint except for a common basepoint $y_{0}$ and whose tangent directions agree at $y_{0}$. We call $\Gamma=\left\{\gamma_{k}\right\}$ a link. A link $\Gamma$ is called mod-2 trivial if $0=$ $\left[\gamma_{k}\right] \in H_{1}\left(Y ; \mathbb{Z}_{2}\right)$ for all $k$. We use the same notation for the "thickened" loops $\gamma_{k}: S^{1} \times D^{2} \rightarrow Y$; the latter should be chosen so as to have a common normal disk at $y_{0}$. Let $\eta(z)$ be a smooth rotationally symmetric bump function on the unit disk $D^{2}$ with support away from the boundary of $D^{2}$ and with integral one. Finally, let $f: S U(2)^{n} \rightarrow \mathbb{R}$ be a smooth function which is invariant with respect to conjugation, that is, $f\left(u g_{1} u^{-1}, \ldots, u g_{n} u^{-1}\right)=f\left(g_{1}, \ldots, g_{n}\right)$ for all $u \in S U(2)$.

Following the construction in Section 3.2, choose a lifting of the holonomy to $S U(2)$. It is uniquely determined by a choice of square roots of hol $C$ on a set of representative loops, different choices leading to equivalent theories. For each based loop $\gamma$, we obtain a well defined map $\mathcal{A}(P) \rightarrow S U(2)$. Define

$$
h(A)=\int_{D^{2}} f\left(\operatorname{hol}_{A}\left(\gamma_{1}\left(S^{1} \times\{z\}\right)\right), \ldots, \operatorname{hol}_{A}\left(\gamma_{n}\left(S^{1} \times\{z\}\right)\right)\right) \eta(z) d^{2} z,
$$

where $\operatorname{hol}_{A}\left(\gamma_{k}\left(S^{1} \times\{z\}\right)\right)$ stands for holonomy of $A$ around the loop $\gamma_{k}\left(S^{1} \times\{z\}\right)$, $z \in D^{2}$, starting at the normal disk at $y_{0}$. The action of $\mathcal{G}(P)$ only changes holonomies around $\gamma_{k}\left(S^{1} \times\{z\}\right)$ within their $S U(2)$ conjugacy class, therefore, we have a well defined function

$$
h: \mathcal{B}(P) \rightarrow \mathbb{R}
$$

which is called an admissible perturbation relative to $\Gamma$. For any link $\Gamma$, denote by $\mathcal{H}_{\Gamma}$ the space of admissible perturbations relative to $\Gamma$. 
Lemma 5.1. If $\Gamma$ is mod-2 trivial then the function $h$ defined in (10) is $H^{1}\left(Y ; \mathbb{Z}_{2}\right)$ invariant.

Proof. We need to prove that $h(A \otimes \chi)=h(A)$ for all $A \in \mathcal{A}(P)$ and $\chi \in H^{1}\left(Y ; \mathbb{Z}_{2}\right)$. This follows easily from the formula

$$
\operatorname{hol}_{A \otimes \chi}\left(\gamma_{k}\left(S^{1} \times\{z\}\right)\right)=\operatorname{hol}_{A}\left(\gamma_{k}\left(S^{1} \times\{z\}\right)\right) \cdot \chi\left(\gamma_{k}\right)
$$

after we notice that $\chi\left(\gamma_{k}\right)=1$ because $\chi: \pi_{1} Y \rightarrow \mathbb{Z}_{2}$ factors through $H_{1}\left(Y ; \mathbb{Z}_{2}\right)$.

Any admissible perturbation $h \in \mathcal{H}_{\Gamma}$ where $\Gamma$ is a mod-2 trivial link will be called an equivariant admissible perturbation.

\subsection{Perturbed projectively flat connections}

Let $h: \mathcal{A}(P) \rightarrow \mathbb{R}$ be an admissible perturbation relative to a link $\Gamma$. The projection map $\pi: \mathcal{A}(P) \rightarrow \mathcal{A}(\bar{P})$ identifies the tangent space of $\mathcal{A}(P)$ with that of $\mathcal{A}(\bar{P})$. Identify the latter with $\Omega^{1}(Y$; ad $\bar{P})$ and define $\zeta_{h}: \mathcal{A}(P) \rightarrow \Omega^{1}(Y$;ad $\bar{P})$ by the formula

$$
\zeta_{h}(A)=* F_{\bar{A}}-4 \pi^{2} \cdot \nabla h(A),
$$

where $\nabla h$ is the $L^{2}$-gradient of $h$. A straightforward calculation shows that, up to the identification of the tangent spaces, $\zeta_{h}$ is just $-4 \pi^{2}$ times the $L^{2}$-gradient of the function $\mathbf{c s}+h$.

A connection $A \in \mathcal{A}(P)$ is called h-perturbed projectively flat if $\zeta_{h}(A)=0$. The moduli space of $h$-perturbed projectively flat connections is denoted by $\mathcal{M}_{h}(P)$ so that $\mathcal{M}_{h}(P)=\zeta_{h}^{-1}(0) / \mathcal{G}(P)$. If $h=0$ then $\mathcal{M}_{h}(P)$ coincides with the moduli space $\mathcal{M}(P)$ of projectively flat connections, see Section 2.2.

Next we wish to describe the local structure of $\mathcal{M}_{h}(P)$ near a point $[A] \in$ $\mathcal{M}_{h}(P)$. The slice through $A$ to the $\mathcal{G}(P)$-action on $\mathcal{A}(P)$ is the affine subspace

$$
X_{A}=\left\{A+\pi_{*}^{-1}(a) \mid a \in \operatorname{ker} d_{\bar{A}}^{*}\right\} \subset \mathcal{A}(P)
$$

where $d_{\bar{A}}^{*}: \Omega^{1}(Y$; ad $\bar{P}) \rightarrow \Omega^{0}(Y$; ad $\bar{P})$. Since $c_{1}(P)$ is an odd element in $H^{2}(Y ; \mathbb{Z})$, the stabilizer of $A$ in $\mathcal{G}(P)$ coincides with the center of $S U(2)$, and a small neighborhood of $A$ in $X_{A}$ gives a local model for $\mathcal{B}(P)$ near $[A]$. Therefore, the moduli space $\mathcal{M}_{h}(P)$ near $[A] \in \mathcal{M}_{h}(P)$ is the zero set of $\zeta_{h}$ restricted to the slice $X_{A}$. The linearization of $\zeta_{h}: \mathcal{A}(P) \rightarrow \Omega^{1}(Y$; ad $\bar{P})$ at $A \in \mathcal{A}(P)$ is the operator

$$
* d_{A, h}=* d_{\bar{A}}-4 \pi^{2} \cdot \operatorname{Hess} h(A): \Omega^{1}(Y ; \operatorname{ad} \bar{P}) \rightarrow \Omega^{1}(Y ; \operatorname{ad} \bar{P}),
$$

hence the tangent space to $\mathcal{M}_{h}(P)$ at $[A] \in \mathcal{M}_{h}(P)$ can be identified with

$$
H_{h}^{1}(Y ; \operatorname{ad} A)=\operatorname{ker} * d_{A, h} / \operatorname{im}\left\{d_{\bar{A}}: \Omega^{0}(Y ; \operatorname{ad} \bar{P}) \rightarrow \Omega^{1}(Y ; \operatorname{ad} \bar{P})\right\} .
$$

We call $\mathcal{M}_{h}(P)$ non-degenerate at $[A] \in \mathcal{M}_{h}(P)$ if $H_{h}^{1}(Y ; \operatorname{ad} A)=0$; we call it non-degenerate if it is non-degenerate at all $[A] \in \mathcal{M}_{h}(P)$. If $\mathcal{M}_{h}(P)$ 
is non-degenerate, it consists of finitely many points, and their signed count gives $\lambda^{\prime \prime \prime}(Y, w)$ where $c_{1}(P)=w(\bmod 2)$.

If $h$ is an equivariant admissible perturbation then according to Lemma 5.1, $\mathcal{M}_{h}(P)$ is acted upon by $H^{1}\left(Y ; \mathbb{Z}_{2}\right)$.

\subsection{Abundance of equivariant admissible perturbations}

Our main goal in the next few sections will be to show that one can always find an equivariant admissible perturbation $h$ such that $\mathcal{M}_{h}(P)$ is non-degenerate. We begin by choosing a mod-2 trivial link $\Gamma$ satisfying certain necessary conditions. Such links are called abundant; the definition of abundance at $A$ depends on the size of the stabilizer of $A$ in $H^{1}\left(Y ; \mathbb{Z}_{2}\right)$.

Let $\Gamma=\left\{\gamma_{k}\right\}$ be a mod-2 trivial link and $A$ a projectively flat connection whose stabilizer in $H^{1}\left(Y ; \mathbb{Z}_{2}\right)$ is trivial. Then $\Gamma$ is called abundant at $A$ if there exist admissible perturbations $h_{i} \in \mathcal{H}_{\Gamma}, i=1, \ldots, m$, such that the map from $\mathbb{R}^{m}$ to $\operatorname{Hom}\left(H^{1}(Y ;\right.$ ad $\left.A), \mathbb{R}\right)$ given by

$$
\left(x_{1}, \ldots, x_{m}\right) \mapsto \sum_{i=1}^{m} x_{i} D h_{i}(A)
$$

is surjective. We will refer to this as 'first order' abundance.

Now, let $A$ be a projectively flat connection whose stabilizer in $H^{1}\left(Y ; \mathbb{Z}_{2}\right)$ equals $\mathbb{Z}_{2}$. Let $\tau$ be a generator in $\mathbb{Z}_{2}$ and denote by $V^{ \pm}(A)$ respectively the $( \pm 1)$-eigenspaces of $\tau_{*}: H^{1}(Y ; \operatorname{ad} A) \rightarrow H^{1}(Y ; \operatorname{ad} A)$. Denote by $\operatorname{Sym}(V)$ the set of symmetric bilinear forms on a vector space $V$. A mod-2 trivial link $\Gamma$ is called abundant at $A$ if there exist admissible perturbations $h_{1}, \ldots, h_{m} \in \mathcal{H}_{\Gamma}$ such that $D h_{k+1}(A)=\ldots=D h_{m}(A)=0$ for some $k$, and the map from $\mathbb{R}^{m}$ to $\operatorname{Hom}\left(V^{+}(A), \mathbb{R}\right) \oplus \operatorname{Sym}\left(V^{-}(A)\right)$ given by

$$
\left(x_{1}, \ldots, x_{m}\right) \mapsto\left(\sum_{i=1}^{k} x_{i} D h_{i}(A), \sum_{i=k+1}^{m} x_{i} \operatorname{Hess} h_{i}(A)\right)
$$

is surjective. This will be referred to as 'second order' abundance.

Due to the fact (cf. Section 4.3) that $H^{1}(Y$; ad $A)=0$ for any projectively flat connection $A$ whose stabilizer is bigger than $\mathbb{Z}_{2}$, we do not need to perturb $A$ and hence do not need the concept of abundance at such a connection.

Note that the property of abundance is preserved by the gauge group action. Another useful remark is that if $\Gamma$ is abundant at $A$, and $\Gamma_{0}$ is another link whose components are sufficiently close to those of $\Gamma$, then $\Gamma_{0}$ is also abundant at $A$. Moreover, the perturbation functions $h_{i}$ can be taken to be the same as for $\Gamma$. These facts come from the homotopy invariance of parallel transport. Note also that if $\Gamma$ is abundant at $A$ and $\Gamma \subset \Gamma^{\prime}$ then $\Gamma^{\prime}$ is also abundant at $A$. The following result will be proved in Section 5.5 below. 
Proposition 5.2. There exists a mod-2 trivial link $\Gamma$ which is abundant at all $[A] \in \mathcal{M}(P)$ whose stabilizer is at most $\mathbb{Z}_{2}$.

\subsection{Non-degeneracy results}

In this section, we will make use of Proposition 5.2 to prove existence of equivariant admissible perturbation functions making $\mathcal{M}_{h}(P)$ non-degenerate.

Let $\Gamma$ be an abundant mod-2 trivial link as in Proposition 5.2 and consider the universal zero set

$$
\mathcal{Z}=\left\{([A], h) \in \mathcal{B}(P) \times \mathcal{H}_{\Gamma} \mid \zeta_{h}(A)=0\right\} .
$$

The moduli space $\mathcal{M}^{*}$ which consists of projectively flat connections with trivial stabilizer in $H^{1}\left(Y ; \mathbb{Z}_{2}\right)$ will be viewed as a subset of $\mathcal{Z}$ by assigning $([A], 0)$ to $[A] \in \mathcal{M}^{*}$. The following proposition roughly asserts that $\mathcal{M}^{*}$ can be "thickened" inside $\mathcal{Z}$ to become a smooth manifold.

Proposition 5.3. The moduli space $\mathcal{M}^{*}$ has an open neighborhood $\mathcal{U}^{*} \subset \mathcal{Z}$ which is a submanifold of $\mathcal{B}(P) \times \mathcal{H}_{\Gamma}$.

Proof. Fix a point $\left[A_{0}\right] \in \mathcal{M}^{*}$ and consider the map

$$
P: X_{A_{0}} \times \mathcal{H}_{\Gamma} \rightarrow \operatorname{ker} d_{\bar{A}_{0}}^{*}
$$

given by $P(A, h)=\Pi_{A_{0}} \zeta_{h}(A)$ where $\Pi_{A_{0}}: \Omega^{1}(Y$; ad $\bar{P}) \rightarrow \operatorname{ker} d_{\bar{A}_{0}}^{*}$ is the $L^{2}$ orthogonal projection. The first partial derivative of this map is Fredholm with cokernel $H^{1}\left(Y\right.$; ad $\left.A_{0}\right)$. Since $\Gamma$ is abundant at $A_{0}$, the image of the partial derivative $\partial P / \partial h$ is a subspace which orthogonally projects onto this cokernel. Therefore, $P$ is a submersion at $\left[A_{0}\right]$. The implicit function theorem now implies that $P^{-1}(0) \subset X_{A_{0}} \times \mathcal{H}_{\Gamma}$ is smooth near $\left(A_{0}, 0\right)$. Moreover, $\Pi_{A_{0}} \zeta_{h}(A)=0$ if and only if $\zeta_{h}(A)=0$, at least in a small neighborhood of $A_{0}$ in $X_{A_{0}}$, see [15, Lemma 12.1.2]. The union of such neighborhoods over all $\left[A_{0}\right] \in \mathcal{M}^{*}$ is the open submanifold $\mathcal{U}^{*}$.

Let us now turn to connections in $\mathcal{B}(P)$ with stabilizer $\mathbb{Z}_{2}$. Fix a generator $\tau$ in a copy of $\mathbb{Z}_{2}$ and consider the subset $\mathcal{B}^{\tau}$ of $\mathcal{B}(P)$ consisting of gauge equivalence classes of connections stabilized by $\tau$. The argument of Proposition 5.3, after a slight modification, can be used to prove that $\mathcal{M}^{\tau}$ has an open manifold neighborhood inside $\mathcal{B}^{\tau}$. Since we are interested in non-degeneracy inside $\mathcal{B}(P)$, we need to study the normal bundle of $\mathcal{M}_{h}^{\tau}$ in $\mathcal{M}_{h}(P)$.

To describe this normal bundle, we will review the Kuranishi model of $\mathcal{M}(P)$ near $[A] \in \mathcal{M}^{\tau}$, see [10]. Since the derivative of $\Pi_{A} \zeta(A): X_{A} \rightarrow \operatorname{ker} d_{\bar{A}}^{*}$ is already a Fredholm isomorphism from the orthogonal complement of its kernel to the orthogonal complement of its cokernel, the effect on the normal bundle of adding a small perturbation $h$ is determined by Hess $h(A): V^{-}(A) \rightarrow V^{-}(A)$. 
In particular, the normal bundle is zero dimensional whenever Hess $h(A)$ is an isomorphism.

Let us consider the universal zero set

$$
\mathcal{Z}^{\tau}=\left\{([A], h) \in \mathcal{B}^{\tau} \times \mathcal{H}_{\Gamma} \mid \zeta_{h}(A)=0\right\}
$$

and view $\mathcal{M}^{\tau}=\mathcal{M}(P) \cap \mathcal{B}^{\tau}$ as a subset of $\mathcal{Z}^{\tau}$ by assigning $([A], 0)$ to $[A] \in \mathcal{M}^{\tau}$.

Proposition 5.4. The moduli space $\mathcal{M}^{\tau}$ has an open neighborhood $\mathcal{U}^{\tau} \subset \mathcal{Z}^{\tau}$ such that

(a) $\mathcal{U}^{\tau}$ is a submanifold in $\mathcal{B}^{\tau} \times \mathcal{H}_{\Gamma}$, and

(b) for every $A$, a generic $h$ such that $([A], h) \in \mathcal{U}^{\tau}$, has non-degenerate Hessian.

Proof. Let us fix $\left[A_{0}\right] \in \mathcal{M}^{\tau}$. The slice at $A_{0}$ of the gauge group action on $\mathcal{B}^{\tau}$ is given by

$$
X_{A_{0}}^{\tau}=\left\{A_{0}+\pi_{*}^{-1}(a) \mid a \in \operatorname{ker} d_{\bar{A}_{0}}^{*} \cap \Omega^{1}(Y ; \operatorname{ad} \bar{P})^{+}\right\},
$$

where $\Omega^{1}(Y \text {; ad } \bar{P})^{+}$is the $(+1)$-eigenspace of $\tau: \Omega^{1}(Y$; ad $\bar{P}) \rightarrow \Omega^{1}(Y ; \operatorname{ad} \bar{P})$. Denote by $\operatorname{Sym}\left(V^{-}\right)$the bundle over an open neighborhood $W$ of $\left(A_{0}, 0\right)$ in $X_{A_{0}}^{\tau} \times$ $\mathcal{H}_{\Gamma}$ whose fiber over $(A, h)$ is $\operatorname{Sym}\left(V_{h}^{-}(A)\right)$, the set of symmetric bilinear forms on the $(-1)$-eigenspace $V_{h}^{-}(A)$ of $\tau: H_{h}^{1}(Y$; ad $A) \rightarrow H_{h}^{1}(Y$; ad $A)$, compare with [4, page 173]. Let

$$
P: W \rightarrow\left(\operatorname{ker} d_{\bar{A}_{0}}^{*} \cap \Omega^{1}(Y ; \operatorname{ad} \bar{P})^{+}\right) \oplus \underline{\operatorname{Sym}}\left(V^{-}\right)
$$

be the section $P(A, h)=\left(\Pi_{A_{0}}^{*} \zeta_{h}(A)\right.$, Hess $\left.h(A)\right)$ where $\Pi_{A_{0}}^{*}$ is $\Pi_{A_{0}}$ followed by the $L^{2}$-orthogonal projection onto $\operatorname{ker} d_{\bar{A}_{0}}^{*} \cap \Omega^{1}(Y ; \text { ad } \bar{P})^{+}$. The first partial derivative of $P$ at $\left(A_{0}, 0\right)$ has cokernel $V^{+}\left(A_{0}\right) \oplus \operatorname{Sym}\left(V^{-}\left(A_{0}\right)\right)$. Since $\Gamma$ is abundant at $A_{0}$, the image of the partial derivative $\partial P / \partial h$ is a subspace which orthogonally projects onto this cokernel. The implicit function theorem now implies that $P^{-1}(\{0\} \times$ $\left.\operatorname{Sym}\left(V^{-}\left(A_{0}\right)\right)\right)$ is smooth near $\left(A_{0}, 0\right)$, which proves part (a). Since non-degenerate symmetric forms are generic in $\operatorname{Sym}\left(V^{-}\left(A_{0}\right)\right)$, the part (b) also follows.

Corollary 5.5. For a small generic admissible perturbation $h \in \mathcal{H}_{\Gamma}$ the moduli space $\mathcal{M}_{h}(P)$ is non-degenerate.

Proof. Since the universal zero set $\mathcal{Z}$ is a smooth manifold near the the moduli space $\mathcal{M}^{*} \cup \mathcal{M}^{\tau}$ the result follows by applying the Sard-Smale theorem to the projection to $\mathcal{H}_{\Gamma}$.

\subsection{Proof of Proposition 5.2}

The proof of Proposition 5.2 naturally divides into three parts, which can be viewed as pointwise, local, and global abundance. The passage from pointwise to 
local and global abundance is proved in essentially the same manner as in [10]. These rely on basic analytical properties of the Chern-Simons function, especially the compactness of the perturbed moduli space, and the restriction to equivariant admissible perturbations does not change these arguments. Thus we will concentrate on establishing pointwise abundance. For connections with trivial stabilizer, this is done in Lemma 5.7, while for connections with stabilizer $\mathbb{Z}_{2}$ in Lemma 5.10.

Denote by $p: \tilde{Y} \rightarrow Y$ the regular covering space corresponding to the surjection $\varphi_{2}: \pi_{1} Y \rightarrow H_{1}\left(Y ; \mathbb{Z}_{2}\right) \cong\left(\mathbb{Z}_{2}\right)^{3}$. This cover might be called the 2 -universal abelian cover, because of the following observation. Let $G$ be an abelian group which is a $\mathbb{Z}_{2}$-vector space, and suppose that $f: \pi_{1} Y \rightarrow G$ is a homomorphism. Then there is a unique homomorphism $\hat{f}: H_{1}\left(Y ; \mathbb{Z}_{2}\right) \rightarrow G$ such that $\hat{f} \circ \varphi_{2}=f$. This can be readily seen from the universal property of the abelianization $\varphi: \pi_{1} Y \rightarrow H_{1}(Y ; \mathbb{Z})$ and the universal property of the map $H_{1}(Y ; \mathbb{Z}) \rightarrow H_{1}(Y ; \mathbb{Z}) \otimes \mathbb{Z}_{2} \cong H_{1}\left(Y ; \mathbb{Z}_{2}\right)$.

For a connection $A$ on the bundle $P \rightarrow Y$, we will denote by $\tilde{A}$ its pull-back to $\tilde{Y}$. We need to understand the behavior of a projectively flat connection on $Y$, when lifted in this manner to $\tilde{Y}$.

Lemma 5.6. Let $\rho: \pi_{1} Y \rightarrow S U(2)$ be a projective representation and $\tilde{\rho}: \pi_{1} \tilde{Y} \rightarrow$ $S U(2)$ the induced projective representation. Let $\operatorname{Stab}(\rho)$ denote the stabilizer of $\rho$ in $H^{1}\left(Y ; \mathbb{Z}_{2}\right)$ then

(a) $\operatorname{Stab}(\rho)=1$ if and only if $\tilde{\rho}$ is irreducible,

(b) $\operatorname{Stab}(\rho)=\mathbb{Z}_{2}$ if and only if $\tilde{\rho}$ is reducible non-central, and

(c) $\operatorname{Stab}(\rho)=\mathbb{Z}_{2} \oplus \mathbb{Z}_{2}$ if and only if $\tilde{\rho}$ is central.

No other stabilizers $\operatorname{Stab}(\rho)$ may occur.

Proof. According to Remark 4.2, the only $\operatorname{Stab}(\rho)$ that occur are $1, \mathbb{Z}_{2}$, and $\mathbb{Z}_{2} \oplus \mathbb{Z}_{2}$.

Suppose that $\operatorname{Stab}(\rho)=\mathbb{Z}_{2} \oplus \mathbb{Z}_{2}$ then, as we saw in the proof of Proposition 4.1, the image of $\rho$ is contained in a copy of the group $Q=\{ \pm 1, \pm i, \pm j, \pm k\}$. Since $\pi_{1} \tilde{Y}$ is in the kernel of the map $\varphi_{2}$, we conclude that the image of $\tilde{\rho}$ is contained in the kernel of the corresponding map $Q \rightarrow H_{1}\left(Q ; \mathbb{Z}_{2}\right) \cong \mathbb{Z}_{2} \oplus \mathbb{Z}_{2}$. This kernel is the same as the commutator subgroup $[Q, Q]=\{ \pm 1\}$ hence $\tilde{\rho}$ is central. Conversely, if $\tilde{\rho}$ is central, its adjoint representation ad $\tilde{\rho}$ is trivial so that $\operatorname{im}(\operatorname{ad} \rho)$ is contained in a subgroup of $S O(3)$ of order at most eight. Therefore, $\operatorname{im}(\operatorname{ad} \rho)$ is contained in a copy of $\mathbb{Z}_{2} \oplus \mathbb{Z}_{2}$, and then $\operatorname{im}(\rho) \subset Q$. In particular, $\operatorname{Stab}(\rho)=\mathbb{Z}_{2} \oplus \mathbb{Z}_{2}$.

Now suppose that $\operatorname{Stab}(\rho)=\mathbb{Z}_{2}$. As in the proof of Proposition 4.1 we see that the image of $\rho$ is contained in a copy of $S_{i} \cup j \cdot S_{i}$ where $S_{i}$ is the unit complex circle. By the argument about the abelianization $\bmod 2$, it follows that $\operatorname{im}(\tilde{\rho}) \subset S_{i}$, so that $\tilde{\rho}$ is abelian. Conversely, if $\tilde{\rho}$ is abelian then $\operatorname{im}(\operatorname{ad} \tilde{\rho})$ is contained in a copy of $S O(2)$, and $\operatorname{im}(\operatorname{ad} \rho)$ in its finite 2-prime extension. Therefore, $\operatorname{im}(\rho)$ is contained in a copy of $S_{i} \cup j \cdot S_{i}$.

The remaining case follows by elimination.

The same result holds for projectively flat connections in place of projective representations. 
Now we are able to deduce the existence of abundant links in the simplest case, when the stabilizer of $A$ in $H^{1}\left(Y ; \mathbb{Z}_{2}\right)$ is trivial.

Lemma 5.7. Let $A$ be a projectively flat connection whose stabilizer in $H^{1}\left(Y ; \mathbb{Z}_{2}\right)$ is trivial. Then there exists a mod-2 trivial link $\Gamma$ that is abundant at $A$.

Proof. By Lemma 5.6, the connection $\tilde{A}$ is irreducible. According to [10, Lemma 60], see also [8, Lemma 2c.1] and [20, Lemma 8.1], there is a link $\tilde{\Gamma}$ in $\tilde{Y}$ that is abundant at $\tilde{A}$. If we perturb $\tilde{\Gamma}$ by a small amount, its projection $\Gamma=p(\tilde{\Gamma})$ will be a link in $Y$. It is clear that $\Gamma$ is mod-2 trivial; we claim that in fact it is abundant. In the discussion that follows, the perturbing functions $h_{i}$ on $Y$ will be the push-down of the perturbing functions $\tilde{h}_{i}$ on $\tilde{Y}$. This makes sense because the holonomy of $\tilde{A}$ around a component $\tilde{\gamma}$ of $\tilde{\Gamma}$ is the same as the holonomy of $A$ around $p(\tilde{\gamma})$.

Consider the commutative diagram

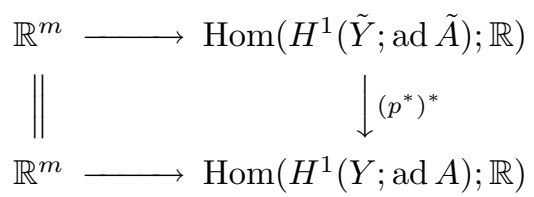

where the horizontal arrows are the holonomy maps as in (11). The arrow along the top is surjective, because $\tilde{\Gamma}$ is abundant at $\tilde{A}$. Now it is a standard consequence of the transfer sequence [5] that the map

$$
p^{*}: H^{1}(Y ; \operatorname{ad} A) \rightarrow H^{1}(\tilde{Y} ; \operatorname{ad} \tilde{A})
$$

is injective. Hence the bottom arrow is surjective as well.

We next turn our attention to the abundance at projectively flat connections with stabilizer $\mathbb{Z}_{2}$ in $H^{1}\left(Y ; \mathbb{Z}_{2}\right)$. Let $\rho: \pi_{1} Y \rightarrow S U(2)$ be a projective representation with $\operatorname{Stab}(\rho)=\mathbb{Z}_{2}$ and fix a generator $\tau \in \mathbb{Z}_{2} \subset H^{1}\left(Y ; \mathbb{Z}_{2}\right)$. Then $\tau$ acts on $\mathcal{P R}_{c}(Y ; S U(2))$ fixing $\rho$ and hence inducing a $\mathbb{Z}_{2}$-action $\tau^{*}$ on the tangent space $T_{\rho} \mathcal{P} \mathcal{R}_{c}(Y ; S U(2))=H^{1}(Y ; \operatorname{ad} \rho)$. Denote as before by $V^{ \pm}(\rho)$ the $( \pm 1)$-eigenspaces of $\tau^{*}$ so that $H^{1}(Y ; \operatorname{ad} \rho)=V^{+}(\rho) \oplus V^{-}(\rho)$.

According to Lemma 5.6 the lift $\tilde{\rho}: \pi_{1} \tilde{Y} \rightarrow S U(2)$ of $\rho$ is a reducible (noncentral) projective representation. Assuming (after conjugation if necessary) that $\operatorname{im}(\tilde{\rho})$ is contained in the complex circle $S_{i}$, we obtain a splitting ad $\tilde{\rho}=\mathbb{R} \oplus \operatorname{ad}_{\mathbb{C}} \tilde{\rho}$ where $\mathbb{R}$ stands for a trivial one-dimensional representation and $\operatorname{ad}_{\mathbb{C}} \tilde{\rho}: \pi_{1} \tilde{Y} \rightarrow$ $S O(2)$. Accordingly, $H^{1}(\tilde{Y}$; ad $\tilde{\rho})$ splits as

$$
H^{1}(\tilde{Y} ; \operatorname{ad} \tilde{\rho})=H^{1}(\tilde{Y} ; \mathbb{R}) \oplus H^{1}\left(\tilde{Y} ; \operatorname{ad}_{\mathbb{C}} \tilde{\rho}\right) .
$$

Lemma 5.8. The projection $p: \tilde{Y} \rightarrow Y$ induces a monomorphism $p^{*}: H^{1}(Y ; \operatorname{ad} \rho)$ $\rightarrow H^{1}(\tilde{Y} ; \operatorname{ad} \tilde{\rho})$ such that

$$
p^{*}\left(V^{+}(\rho)\right) \subset H^{1}(\tilde{Y} ; \mathbb{R}) \quad \text { and } \quad p^{*}\left(V^{-}(\rho)\right) \subset H^{1}\left(\tilde{Y} ; \operatorname{ad}_{\mathbb{C}} \tilde{\rho}\right) .
$$


Proof. That $p^{*}$ is a monomorphism follows from the standard transfer argument, see [5]. Since the conjugacy class of $\rho$ is fixed by $\tau$, there exists an element $u \in S U(2)$ such that $u^{2}=-1$ and $\tau(x) \rho(x)=u \rho(x) u^{-1}$ for all $x \in \pi_{1} Y$. If $x \in \pi_{1} \tilde{Y}$ then $\tilde{\rho}(x)=\rho(x)$ and $\tau(x)=1$ so that $\tilde{\rho}(x)=u \tilde{\rho}(x) u^{-1}$. Since $\tilde{\rho}(x) \in S_{i}$ we conclude that $u= \pm i$.

To describe the induced action $\tau^{*}$ on $T_{\rho} \mathcal{P} \mathcal{R}_{c}(Y ; S U(2))=H^{1}(Y$; ad $\rho)$ we first identify the tangent spaces at $\rho$ and $\rho^{\tau}$ by ad $u$, and then linearize the map $\rho \mapsto \rho^{\tau}$ as follows:

$$
\begin{aligned}
& (1+\varepsilon \cdot \xi(x)) \rho(x) \stackrel{\tau}{\rightarrow} \\
& \qquad(x)(1+\varepsilon \cdot \xi(x)) \rho(x)=(1+\varepsilon \cdot \xi(x)) \tau(x) \rho(x) \stackrel{\operatorname{ad} u}{\longrightarrow} \\
& \quad u(1+\varepsilon \cdot \xi(x)) \tau(x) \rho(x) u^{-1}=\left(1+\varepsilon \cdot u \xi(x) u^{-1}\right) \rho(x) .
\end{aligned}
$$

Here, $\xi: \pi_{1} Y \rightarrow \mathfrak{s u}(2)$ is a 1-cocycle representing an element of $H^{1}(Y$; ad $\rho)$, and $\varepsilon$ is a small positive real number. Thus the action $\tau^{*}: H^{1}(Y$; ad $\rho) \rightarrow H^{1}(Y$; ad $\rho)$ at the level of 1-cocycles is given by the formula $\tau^{*}(\xi)=u \xi u^{-1}$. Since $u= \pm i$, the subspace $V^{+}(\rho)$ is generated by 1-cocycles $\xi$ with $\operatorname{im}(\xi) \subset i \mathbb{R}$, and $V^{-}(\rho)$ by 1-cocycles $\xi$ with $\operatorname{im}(\xi)$ in the subspace $\mathbb{C} \subset \mathfrak{s u}(2)$ spanned by $j$ and $k$.

The embedding $p^{*}: H^{1}(Y$; ad $\rho) \rightarrow H^{1}(\tilde{Y}$; ad $\tilde{\rho})$ is given by pulling back the 1-cocycles $\xi: \pi_{1} Y \rightarrow \mathfrak{s u}(2)$ via the homomorphism $p_{*}: \pi_{1} \tilde{Y} \rightarrow \pi_{1} Y$. In particular, if $\operatorname{im}(\xi) \subset i \mathbb{R}$ then $\operatorname{im}\left(p^{*} \xi\right) \subset i \mathbb{R}$ so that $\left[p^{*} \xi\right] \in H^{1}(\tilde{Y} ; \mathbb{R})$. Similarly, if $\operatorname{im}(\xi)$ belongs to $\mathbb{C}$ spanned by $j$ and $k$ then $\left[\operatorname{im}\left(p^{*} \xi\right)\right] \in H^{1}\left(\tilde{Y} ; \operatorname{ad}_{\mathbb{C}} \tilde{\rho}\right)$.

Denote by $\tilde{\tau}: \tilde{Y} \rightarrow \tilde{Y}$ the covering transformation corresponding to the (dual of) $\tau \in \mathbb{Z}_{2} \subset H^{1}\left(Y ; \mathbb{Z}_{2}\right)$, and by $\tilde{\tau}^{*}: H^{1}(\tilde{Y}$; ad $\tilde{\rho}) \rightarrow H^{1}(\tilde{Y}$; ad $\tilde{\rho})$ the induced action.

Lemma 5.9. The subset $p^{*}\left(V^{+}(\rho)\right) \subset H^{1}(\tilde{Y} ; \mathbb{R})$ is the $(+1)$-eigenspace of $\tilde{\tau}^{*}$ : $H^{1}(\tilde{Y} ; \mathbb{R}) \rightarrow H^{1}(\tilde{Y} ; \mathbb{R})$, and $p^{*}\left(V^{-}(\rho)\right) \subset H^{1}\left(\tilde{Y} ; \operatorname{ad}_{\mathbb{C}} \tilde{\rho}\right)$ the $(+1)$-eigenspace of $\tilde{\tau}^{*}: H^{1}\left(\tilde{Y} ; \operatorname{ad}_{\mathbb{C}} \tilde{\rho}\right) \rightarrow H^{1}\left(\tilde{Y} ; \operatorname{ad}_{\mathbb{C}} \tilde{\rho}\right)$. Moreover, $p^{*}\left(V^{-}(\rho)\right)$ is a totally real subspace of the complex vector space $H^{1}\left(\tilde{Y} ; \operatorname{ad}_{\mathbb{C}} \tilde{\rho}\right)$.

Proof. The first two statements follow from the standard transfer argument, see [5]. For the last statement, note that the pullback of $\operatorname{ad}_{\mathbb{C}} \tilde{\rho}$ via $\tilde{\tau}$ is exactly the complex conjugate representation $\overline{\operatorname{ad}_{\mathbb{C}} \tilde{\rho}}$. It follows that the action of $\tilde{\tau}^{*}$ on the cochains used to compute $H^{1}\left(\tilde{Y} ; \operatorname{ad}_{\mathbb{C}} \tilde{\rho}\right)$ is complex anti-linear, and so the action on this cohomology group is also complex anti-linear. Thus the $(+1)$-eigenspace $p^{*}\left(V^{-}(\rho)\right)$ is totally real.

Lemma 5.10. Let $A$ be a projectively flat connection whose stabilizer in $H^{1}\left(Y ; \mathbb{Z}_{2}\right)$ is $\mathbb{Z}_{2}$. Then there exists a mod-2 trivial link $\Gamma$ that is abundant at $A$.

Proof. By Lemma 5.6, the pull back connection $\tilde{A}$ and the associated projective representation $\tilde{\rho}$ are reducible and non-central. By [10, Corollary 64 and Corollary 
66], see also [4, Proposition 3.4] and [11, Proposition 57], there is a link $\tilde{\Gamma}$ in $\tilde{Y}$ and admissible perturbations $\tilde{h}_{i}: \mathcal{B}(\tilde{P}) \rightarrow \mathbb{R}, i=1, \ldots, m$, such that $D \tilde{h}_{k+1}(A)=$ $\ldots=D \tilde{h}_{m}(A)=0$ for some $k$ and the map

$$
\mathbb{R}^{m} \rightarrow \operatorname{Hom}\left(H^{1}(\tilde{Y} ; \mathbb{R}), \mathbb{R}\right) \oplus \operatorname{Herm}\left(H^{1}\left(\tilde{Y} ; \operatorname{ad}_{\mathbb{C}} \tilde{\rho}\right)\right)
$$

given by

$$
\left(x_{1}, \ldots, x_{m}\right) \mapsto\left(\sum_{i=1}^{k} x_{i} D \tilde{h}_{i}(\tilde{A}), \sum_{i=k+1}^{m} x_{i} \operatorname{Hess} \tilde{h}_{i}(\tilde{A})\right)
$$

is surjective. Here, $\operatorname{Herm}(V)$ stands for the Hermitian forms on a complex vector space $V$. If we perturb $\tilde{\Gamma}$ by a small amount, its projection $\Gamma=p(\tilde{\Gamma})$ will be a link in $Y$. It is clear that $\Gamma$ is mod-2 trivial; we claim that in fact it is abundant. In the discussion that follows, the perturbing functions $h_{i}$ on $Y$ will be the push-down of the perturbing functions $\tilde{h}_{i}$ on $\tilde{Y}$. This makes sense because the holonomy of $\tilde{A}$ around a component $\tilde{\gamma}$ of $\tilde{\Gamma}$ is the same as the holonomy of $A$ around $p(\tilde{\gamma})$.

According to Lemma 5.8, we have the following commutative diagram

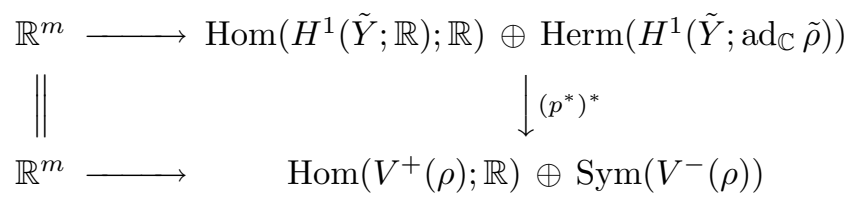

where the horizontal arrows are the holonomy maps as in (12). The arrow along the top is surjective. According to Lemma 5.8, both the map $V^{+}(\rho) \rightarrow H^{1}(\tilde{Y} ; \mathbb{R})$ and the map $V^{-}(\rho) \rightarrow H^{1}\left(\tilde{Y} ; \operatorname{ad}_{\mathbb{C}} \tilde{\rho}\right)$ are injective. By Lemma 5.9, the map

$$
V^{-}(\rho) \rightarrow H^{1}\left(\tilde{Y} ; \operatorname{ad}_{\mathbb{C}} \tilde{\rho}\right)
$$

is obtained by complexification. Therefore, the right arrow in the diagram is surjective, and hence so is the arrow on the bottom.

\subsection{Concluding remarks}

It should be pointed out that there are two slightly differing approaches to the use of holonomy perturbations. While Floer [8] uses perturbations defined in terms of loops with a common basepoint, Taubes and Donaldson [20, 7] use disjoint loops. Note that both perturbation classes are admissible in that they have the right Fredholm properties, and that each of them contains enough perturbations to be 'first order' abundant. Moreover, the larger class of perturbations built from both types of loops is also admissible and 'first order' abundant. Therefore, all of the above perturbation classes lead to equivalent theories, as long as these theories do not involve 'second order' abundance.

In our approach, the 'second order' abundance is only needed when we deal with projectively flat connections with non-trivial stabilizers (in the equivariant 
setting) and reducible projectively flat connections (in the non-equivariant setting). As C. Herald points out in [11] (thus correcting his earlier paper [10]), the use of loops with a common basepoint in this situation is essential.

\section{Proof of Theorem 1.1}

Let $0 \neq w \in H^{2}\left(Y ; \mathbb{Z}_{2}\right)$ and consider a $U(2)$-bundle $P$ with $c_{1}(P)=w(\bmod 2)$. If $w$ is not in the image of $\iota: H^{2}\left(\pi_{1} Y ; \mathbb{Z}_{2}\right) \rightarrow H^{2}\left(Y ; \mathbb{Z}_{2}\right)$ then the theorem follows from Corollary 3.12. Otherwise, choose a 2-cocycle $c$ so that $[c]=w$ and identify $\mathcal{M}(P)$ with $\mathcal{P R}_{c}(Y ; S U(2))$.

If $\mathcal{M}(P)$ is non-degenerate then Theorem 1.1 follows because no orbit in $\mathcal{P} \mathcal{R}_{c}(Y ; S U(2))$ consists of one element, see Remark 4.2, the contribution of the two-orbits equals $\left(a_{1} \cup a_{2} \cup a_{3}\right)[Y](\bmod 2)$ according to Proposition 4.3, and the orbits consisting of four and eight elements do not contribute to $\lambda^{\prime \prime \prime}(Y, w)(\bmod 2)$ at all.

In general, $\mathcal{M}(P)$ needs to be perturbed to make it non-degenerate. The twoorbits are already non-degenerate and hence if our perturbation $h$ is sufficiently small they will remain such. The perturbation $h$ will not create orbits with one element or new orbits with two elements. Moreover, one can always achieve the non-degeneracy by using perturbations which are invariant with respect to the action of $H^{1}\left(Y ; \mathbb{Z}_{2}\right)$, see Corollary 5.5. Therefore, the above argument, discarding the orbits with more than two elements, can be applied again to complete the proof of Theorem 1.1.

\section{The Casson and Rohlin invariants for integral homology spheres}

In this section we explain how our Theorem 1.1 implies Casson's original result that $\lambda(\Sigma)=\rho(\Sigma)(\bmod 2)$ for integral homology spheres $\Sigma$.

\subsection{Calculating the Casson invariant}

Every integral homology sphere $\Sigma$ can be obtained from $S^{3}$ by surgery on an algebraically split link, that is, a link $k_{1} \cup \ldots \cup k_{n}$ such that $\operatorname{lk}\left(k_{i}, k_{j}\right)=0$ for $i \neq j$. Moreover, all the surgery coefficients can be chosen to be 1 or -1 , so that

$$
\Sigma=S^{3}+\varepsilon_{1} \cdot k_{1}+\ldots+\varepsilon_{n} \cdot k_{n}, \quad \varepsilon_{i}= \pm 1 .
$$

The Casson invariants of $\Sigma$ and $\Sigma \pm k$ are related by Casson's surgery formula

$$
\lambda(\Sigma \pm k)=\lambda(\Sigma) \pm \lambda^{\prime}(\Sigma+0 \cdot k),
$$

where $\Sigma+0 \cdot k$ is the result of 0 -surgery of $\Sigma$ along $k$. In Casson's original approach, the term $\lambda^{\prime}$ was interpreted in terms of the Alexander polynomial of the knot $k$. 
For our purposes, we interpret it gauge-theoretically.

Namely, let $P$ be a $U(2)$-bundle over $\Sigma+0 \cdot k$ such that $w_{2}(\bar{P})$ is dual to $[k] \in H_{1}\left(\Sigma+0 \cdot k ; \mathbb{Z}_{2}\right)$. Then $\lambda^{\prime}(\Sigma+0 \cdot k)$ is half a signed count of projectively flat connections in $P$ with a fixed central part, modulo the gauge group consisting of automorphisms of $P$ with determinant one (perhaps after a perturbation). These projectively flat connections are counted with signs determined by the Floer index. Therefore, the invariant $\lambda^{\prime}(\Sigma+0 \cdot k)$ equals half the Euler characteristic of the Floer homology $I_{*}(\Sigma+0 \cdot k)$ so that the surgery formula (14) can be viewed as an application of the Floer exact triangle

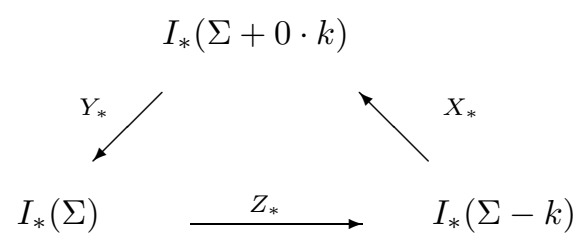

(and similarly for $\lambda(\Sigma+k)$ ). The surgery formula evaluates $\lambda(\Sigma \pm k)$ in terms of $\lambda(\Sigma)$. Surgering out one knot at a time in the surgery presentation (14), we end up with $S^{3}$ whose Casson invariant is known to be trivial.

In order to calculate $\lambda(\Sigma)$ using this approach we need to know the invariants $\lambda^{\prime}(\Sigma+0 \cdot k)$ at each of the steps. To this end, we use another surgery formula

$$
\lambda^{\prime}(\Sigma+0 \cdot k \pm \ell)=\lambda^{\prime}(\Sigma+0 \cdot k) \pm \lambda^{\prime \prime}(\Sigma+0 \cdot k+0 \cdot \ell)
$$

where $k \cup \ell$ is an algebraically split link in $\Sigma$ (it is sufficient to work with algebraically split links because such is the link in presentation (14)). The term $\lambda^{\prime \prime}$ here is defined exactly as $\lambda^{\prime}$ with only difference that now $P$ is a $U(2)$-bundle such that $w_{2}(\bar{P})$ is dual to $[k]+[\ell] \in H_{1}\left(\Sigma+0 \cdot k+0 \cdot \ell ; \mathbb{Z}_{2}\right)$. Again, the above surgery formula follows from the Floer exact triangle, see [3].

This reduces calculation of $\lambda(\Sigma)$ to that of the $\lambda^{\prime \prime}$-invariants. Applying the surgery formula yet another time, we reduce the latter calculation to identifying $\lambda^{\prime \prime \prime}(Y, w)$ for the homology torus $Y$ obtained by 0 -surgery on an algebraically split link $k \cup \ell \cup m$ in $\Sigma$ with $w$ dual to $[k]+[\ell]+[m] \in H_{1}\left(Y ; \mathbb{Z}_{2}\right)$. Theorem 1.1 tells us that $\lambda^{\prime \prime \prime}(Y, w)$ equals $\left(a_{1} \cup a_{2} \cup a_{3}\right)[Y](\bmod 2)$ for any choice of basis $a_{1}, a_{2}$, $a_{3} \in H^{1}\left(Y ; \mathbb{Z}_{2}\right)$.

Remark 7.1. A caveat in the above argument is that the simplification scheme it is based upon fails for computing $\lambda^{\prime}\left(S^{3}+0 \cdot k\right)$. After we simplified $\Sigma$ to $S^{3}$, a new scheme is needed to simplify the knot, not the manifold itself. Such a simplification scheme, based on skein moves, can be found in [1] or [19]. Again, it reduces calculation of $\lambda^{\prime}\left(S^{3}+0 \cdot k\right)$ to that of $\lambda^{\prime \prime \prime}(Y, w)$. 


\subsection{Calculating the Rohlin invariant}

To conclude that $\lambda(\Sigma)=\rho(\Sigma)(\bmod 2)$ for all integral homology spheres $\Sigma$, we will show that the Rohlin invariant satisfies the same surgery formulas as the Casson invariant, only reduced modulo 2 . Results similar to those in this section were found earlier by Turaev [21].

Lemma 7.2. Let $\rho^{\prime}(\Sigma+0 \cdot k)$ be the sum, over the two spin structures on $\Sigma+0 \cdot k$, of their Rohlin invariants. Then $\rho(\Sigma+k)=\rho(\Sigma)+\rho^{\prime}(\Sigma+0 \cdot k)(\bmod 2)$.

Proof. The manifold $\Sigma+0 \cdot k$ can be obtained by 0 -surgery on both $\Sigma+k$ and $\Sigma$. Let $W_{1}$ and $W_{2}$ be the traces of these surgeries, that is, smooth 4-manifolds obtained from $[0,1] \times(\Sigma+k)$, respectively, $[0,1] \times \Sigma$, by attaching a 2-handle along $\{1\} \times k$ with zero framing. Then $W_{1}$ is a spin cobordism between $\Sigma+k$ and $\Sigma+0 \cdot k$ with one spin structure, and $W_{2}$ is a spin cobordism between $\Sigma$ and $\Sigma+0 \cdot k$ with the other spin structure. Since the intersection forms of both $W_{1}$ and $W_{2}$ are zero, we are finished.

Before we continue, note that changing the surgery coefficient from plus to minus does not affect the Rohlin invariant. Therefore, we will assume for the sake of simplicity that all the surgery coefficients $\varepsilon_{i}$ in (14) are equal to one.

Let $k \cup \ell$ be an algebraically split link in $\Sigma$ and define $\rho^{\prime \prime}(\Sigma+0 \cdot k+0 \cdot \ell)$ as the sum, over the four spin structures on $\Sigma+0 \cdot k+0 \cdot \ell$, of their Rohlin invariants. An argument similar to that of Lemma 7.2 proves the surgery formula

$$
\rho^{\prime}(\Sigma+0 \cdot k+\ell)=\rho^{\prime}(\Sigma+0 \cdot k)+\rho^{\prime \prime}(\Sigma+0 \cdot k+0 \cdot \ell)
$$

compare with (15), and yet another application of the same argument yields the formula

$$
\rho^{\prime \prime}(\Sigma+0 \cdot k+0 \cdot \ell)=\rho(\Sigma+k+\ell)+\rho(\Sigma+k)+\rho(\Sigma+\ell)+\rho(\Sigma) .
$$

This reduces the calculation of $\rho(\Sigma)$ to that of the $\rho^{\prime \prime}$-invariants. Applying the surgery formula one more time, we reduce the latter calculation to identifying $\rho^{\prime \prime \prime}(Y)$ for a homology torus $Y=\Sigma+0 \cdot k+0 \cdot \ell+0 \cdot m$. An argument similar to that of Lemma 7.2 yields

$$
\begin{aligned}
\rho^{\prime \prime \prime}(Y)=\rho(\Sigma+k+\ell+m)+\rho(\Sigma+\ell+m)+\rho(\Sigma+k+m) & \\
& +\rho(\Sigma+k+\ell)+\rho(\Sigma+k)+\rho(\Sigma+\ell)+\rho(\Sigma+m)+\rho(\Sigma),
\end{aligned}
$$

which equals $\left(a_{1} \cup a_{2} \cup a_{3}\right)[Y](\bmod 2)$ for any choice of basis $a_{1}, a_{2}, a_{3} \in$ $H^{1}\left(Y ; \mathbb{Z}_{2}\right)$, see [12, Lemma 6.3]. This proves that $\lambda^{\prime \prime \prime}(Y, w)=\rho^{\prime \prime \prime}(Y)(\bmod 2)$ and therefore completes the proof of the formula $\lambda(\Sigma)=\rho(\Sigma)(\bmod 2)$. 


\section{References}

[1] S. Akbulut and J. McCarthy, Casson's invariant for oriented homology 3-spheres. An exposition, Princeton University Press, Princeton, 1990.

[2] M. F. Atiyah and R. Bott, The Yang-Mills equations over Riemann surfaces, Philos. Trans. Roy. Soc. London Ser. A 308 (1983), 523-615.

[3] P. J. Braam and S. K. Donaldson, Floer's work on instanton homology, knots and surgery, in: The Floer memorial volume, Birkhäuser, Basel, 1995, 195-256.

[4] H. Boden and C. Herald, The SU(3) Casson invariant for integral homology 3-spheres, $J$. Diff. Geom. 50 (1998), 147-206.

[5] K. Brown, Cohomology of groups. Springer-Verlag, New York, 1994.

[6] S. K. Donaldson, Topological field theories and formulae of Casson and Meng-Taubes, in: Proceedings of the Kirbyfest (Berkeley, CA, 1998), 87-102 (electronic), Geom. Topol. Publ., Coventry, 1999. URL: http://www.maths.warwick.ac.uk/gt/GTMon2/paper4.abs.html

[7] S. K. Donaldson, Floer homology groups in Yang-Mills theory, Cambridge University Press, Cambridge, 2002.

[8] A. Floer, An instanton-invariant for 3-manifolds, Comm. Math. Phys. 118 (1988), 215-240.

[9] A. Floer, Instanton homology and Dehn surgery, in: The Floer memorial volume, Birkhäuser, Basel, 1995, 77-97.

[10] C. Herald, Legendrian cobordism and Chern-Simons theory on 3-manifolds with boundary, Comm. Anal. Geom. 2 (1994), 337-413.

[11] C. Herald, Transversality for equivariant gradient systems and gauge theory on 3-manifolds, Preprint, 2003.

[12] S. J. Kaplan, Constructing framed 4-manifolds with given almost framed boundaries, Trans. Amer. Math. Soc. 254 (1979), 237-263.

[13] T. T. Q. Le, J. Murakami and T. Ohtsuki, On a universal perturbative invariant of 3manifolds, Topology 37 (1998), 539-574.

[14] C. Lescop, Global surgery formula for the Casson-Walker invariant, Princeton University Press, Princeton, 1996.

[15] J. Morgan, T. Mrowka and D. Ruberman, The $L^{2}$-moduli space and a vanishing theorem for Donaldson polynomial invariants, International Press, 1994.

[16] T. Ohtsuki, Finite type invariants of integral homology 3-spheres, J. Knot Theory Ramifications 5 (1996), 101-115.

[17] D. Ruberman and N. Saveliev, Rohlin's invariant and gauge theory, II. Mapping tori, Geometry and Topology 8 (2004), 35-76. URL: http://arxiv.org/math.GT/0306188.

[18] D. Ruberman and N. Saveliev, Rohlin's invariant and gauge theory, III. Homology 4-tori (submitted). URL: http://arxiv.org/math.GT/0404162.

[19] N. Saveliev, Lectures on the topology of 3-manifolds. An introduction to the Casson invariant, Walter de Gruyter \& Co., Berlin, 1999.

[20] C. Taubes, Casson's invariant and gauge theory, J. Diff. Geom. 31 (1990), 547-599.

[21] V. G. Turaev, Cohomology rings, linking coefficient forms and invariants of spin structures in three-dimensional manifolds, Mat. Sbornik (N.S.) 120(162) (1983), no. 1, 68-83.

[22] K. Walker, An extension of Casson's invariant, Princeton University Press, Princeton, 1992.

D. Ruberman

Department of Mathematics, MS 050

Brandeis University

Waltham, MA 02454

USA

e-mail: ruberman@brandeis.edu
N. Saveliev

Department of Mathematics

University of Miami

PO Box 249085

Coral Gables, FL 33124

USA

e-mail: saveliev@math.miami.edu

(Received: February 13, 2003) 